\title{
Dynamic Response of Composite Lining Tunnel with Buffer Layer: An Analytical and Experimental Investigation
}

\author{
Kaixiang Fan, ${ }^{1}$ Yusheng Shen ${ }^{10},{ }^{1,2}$ Shuaishuai Wang, ${ }^{1,3}$ Bo Gao, ${ }^{1}$ Qing Zheng, \\ Gaoming Yan, ${ }^{1}$ and Peng Min ${ }^{1}$ \\ ${ }^{1}$ Key Laboratory of Transportation Tunnel Engineering, Ministry of Education, School of Civil Engineering, \\ Southwest Jiaotong University, Chengdu 610031, China \\ ${ }^{2}$ National Engineering Laboratory for Technology of Geological Disaster Prevention in Land Transportation, \\ Southwest Jiaotong University, Chengdu 611756, China \\ ${ }^{3}$ CCCC Second Highway Engineering Co., Ltd., Xi'an 710065, China
}

Correspondence should be addressed to Yusheng Shen; sys1997@163.com

Received 16 August 2020; Revised 28 September 2020; Accepted 9 November 2020; Published 23 November 2020

Academic Editor: Kaiming Bi

Copyright (C) 2020 Kaixiang Fan et al. This is an open access article distributed under the Creative Commons Attribution License, which permits unrestricted use, distribution, and reproduction in any medium, provided the original work is properly cited.

Composite lining is often designed for the mountainous tunnels in high-intensity earthquake areas. The application of the buffer layer will bring more advantages, while the shock-absorbing mechanism is still unclear currently. In this paper, based on the Fourier-Bessel series expansion method, the dynamic stress concentration factor of composite lining tunnel with buffer layer subjected to plane SV waves in the half-space is obtained. Then, the influence of geometric and mechanical parameters of the buffer layer on composite lining was systematically analyzed. Finally, the correctness of the analytical solutions is verified by series shaking table tests and numerical simulations. Results suggest that the buffer layer can play the role of "redistributing" the seismic load, and it can effectively reduce the dynamic responses of secondary lining but amplify in primary support. There is an optimal interval of the stiffness and thickness for the buffer layer. When the stiffness ratio of the buffer layer to surrounding rock is $1 / 10 \sim 1 / 50$ or the ratio of buffer layer thickness to inner diameters of secondary lining is $1 / 40 \sim 1 / 20$, the shockabsorbing performance is remarkable. The general damage observations in tests show that the crown, arch springing, and invert of composite lining in case of no buffer layer are prone to cracking under a strong earthquake. The invert of the composite lining is more susceptible to be damaged after adopting the buffer layer. In general, the analytical results were consistent with experimental and numerical results. The above study results may provide theoretical support and experimental data for the seismic design of composite lining tunnels.

\section{Introduction}

Generally, the underground structure has been considered to have better seismic performance compared with surface structure. However, regarding the Kobe earthquake in Japan in 1995, a large number of underground structures suffered severe damage [1], which overturns the traditional concepts. Underground facilities are often used as lifeline engineering, the repair of earthquake damage comes at a high cost, and seismic fortification measures have drawn great attention [2].

Generally, antiseismic and shock absorption measures are two kinds for seismic design of tunnel [3]. The antiseismic measures are mainly to strengthen the surrounding rock (grouting around the lining, drainage, installation of anchor rods, etc.) or to improve the stiffness of the lining structure (using high-performance concrete, increasing the thickness, increasing the reinforcement, etc.) $[4,5]$. The shock absorption measures are usually to design buffer layers with thinner thickness and smaller stiffness around the outer lining. The most common buffer layers are simple plate-type and injection-type; the former is represented by rubber, while the latter is represented by foamed concrete, polymer [6-11]. The buffer layer is much softer than the surrounding rock and lining; thus, the interaction 
between the lining and surrounding rock could be improved $[12,13]$. Compared with antiseismic measures, it is simple and effective to adopt buffer layers [14]. Surely, antiseismic measures and shock absorption measures can both be adopted in the seismic design of tunnels, under some special adverse geological conditions [15]. In practical engineering, the buffer layer mainly consists of rubber material and compressible foamed concrete. Rubber material is a typical superelastic material, its shear modulus is lower than the lining, and it can bear large deformation. Foam concrete is relatively widely used for the following reasons [16]: (1) foamed concrete has good deformation performance due to the presence of a large number of pores; (2) the mix ratio and casting thickness of foamed concrete can be adjusted quantitatively; (3) foamed concrete is easy to adapt to the geological environment and site construction requirements.

Different kinds of methods have been employed for seismic design of tunnels, such as field prototype observation [17-19], shaking table tests [20-24], numerical simulations [25-29], and analytical methods. Field prototype observation data for earthquake damage are scarce. Therefore, the mechanism and measures of antiseismic and shock absorption are mainly explored by analytical methods, shaking table tests, and numerical simulations. Analytical methods play an irreplaceable role compared with the numerical method; besides, it is also an important means to verify the accuracy of numerical methods [30]. Shaking table test can reproduce various seismic waves in the lab, directly reappear the earthquake damage phenomenon, and obtain the dynamic response of the structure, so it is an effective method to solve the complex seismic engineering problems [31].

In terms of analytical methods, wave function expansion has been widely used in the dynamic stress concentration of tunnel structures, which can reveal the mechanical response mechanism of composite lining tunnel with buffer layer in essence and can be used to verify the numerical calculation and test results. Separation of variables is adopted to get the ordinary differential equations in the Fourier-Bessel series expansion method, then the general solutions can be expanded into the Fourier-Bessel series, and finally, the closed solution of the potential function can be solved in given boundary conditions. The key to wave function expansion method is to establish a special coordinate system that can describe the geometry of the scatterer, so it is only applicable to some simple and regular geometry, such as circular, elliptic, or parabolic cross section. In addition, the wave function expansion method is suitable for low-frequency incident wave in the near field. Pao [32] pioneeringly studied the problem of dynamic stress concentration of hollow cavities and lining tunnels in infinite space under elastic wave incidence. Davis [33] and Lee [30, 34-36] deduced the dynamic stress concentration of circular lining tunnel under SH and SV waves incidence and analyzed the dynamic response law of flexible pipe subjected to real seismic wave. Xu et al. [37] gave the series solution of a semispace circular tunnel under subjected to $\mathrm{P}$ waves. Kara [38] studied the diffraction of plane $\mathrm{SH}$ waves in a cylindrical tunnel in a homogeneous and isotropic elastic 1/4 space. Lin [39] proposed analytical solutions for semispace surfaces on stress-free boundary conditions and relaxed boundary conditions. Fan [40] introduced a linear spring model to describe the imperfect contact surface between rock mass and lining and then studied the dynamic response of circular lining tunnel to plane SV waves.

However, most of the literature focuses on monolayer lining and lacking theoretical analysis on the dynamic response of the composite lining. What is more, there is a general lack of experimental demonstration, which makes analytical solutions difficult to apply widely. Hence, in this paper, the wave function expansion method is adopted to study the dynamic stress concentration of composite lining tunnel with buffer layer subjected to plane SV waves at different incident angles in elastic half-space. The influence of Young's modulus and thickness of the buffer layer on the dynamic stress of the composite lining is systematically analyzed. First, the series solutions of stress and displacement of composite lining are obtained by the Fourier-Bessel series expansion. Then, the linear equations of wave function coefficients are solved by MATLAB software, and the dynamic stress concentration factors (DSCF) of composite lining are obtained. The influences of angle of incidence, Young's modulus, and thickness of the buffer layer on DSCF are discussed. Finally, the correctness and reliability of the analytical solution are verified by shaking table tests and numerical simulation. Such studies could provide a reference for the seismic design of shallow-buried composite lining tunnel in high-intensity area.

\section{Model of Composite Lining Tunnel with Buffer Layer}

The model of "Primary Support-Buffer Layer-Secondary Lining" for composite lining tunnel in half-space is depicted in Figure 1.

The distance between the tunnel center and the halfspace surface is $h$. The outer radius of primary support, buffer layer, and the secondary lining is $R_{1}, R_{2}$, and $R_{3}$ respectively. The inner radius of the secondary lining is $R_{4}$. The half-space and the composite lining are assumed to be isotropic, homogeneous, and elastic. The mechanical parameters are determined by Ramey's constants $\lambda_{i}, G_{i}$ and density $\rho_{i}$, and the compression and shear wave velocities are denoted by $c_{\alpha, i}$ and $c_{\beta, i}$, where $i=s, p, b, l$, respectively, representing the surrounding rock, primary support, buffer layer, and secondary lining.

The equations of the scattering waves in the rectangular coordinate system are complicated. To simplify, a large arc is adopted to simulate the surface of half-space; then the scattering waves in half-space can be transformed from rectangular coordinate $o$ to polar coordinate $o_{2}$. The large arc radius $b=10000 R_{1}$, where the center is located at $o_{2}$ and $D$ is the distance between $o_{1}$ and $o_{2}$. Studies indicate that the solution converges to the exact solution when the arc is large enough [30]. In this section, the influence of initial stress is not considered in theoretical analysis, and the contact between surrounding rock and tunnel is ignored. It is assumed that the stress and displacement are continuous at the interfaces between the composite lining tunnel and the half-space. 


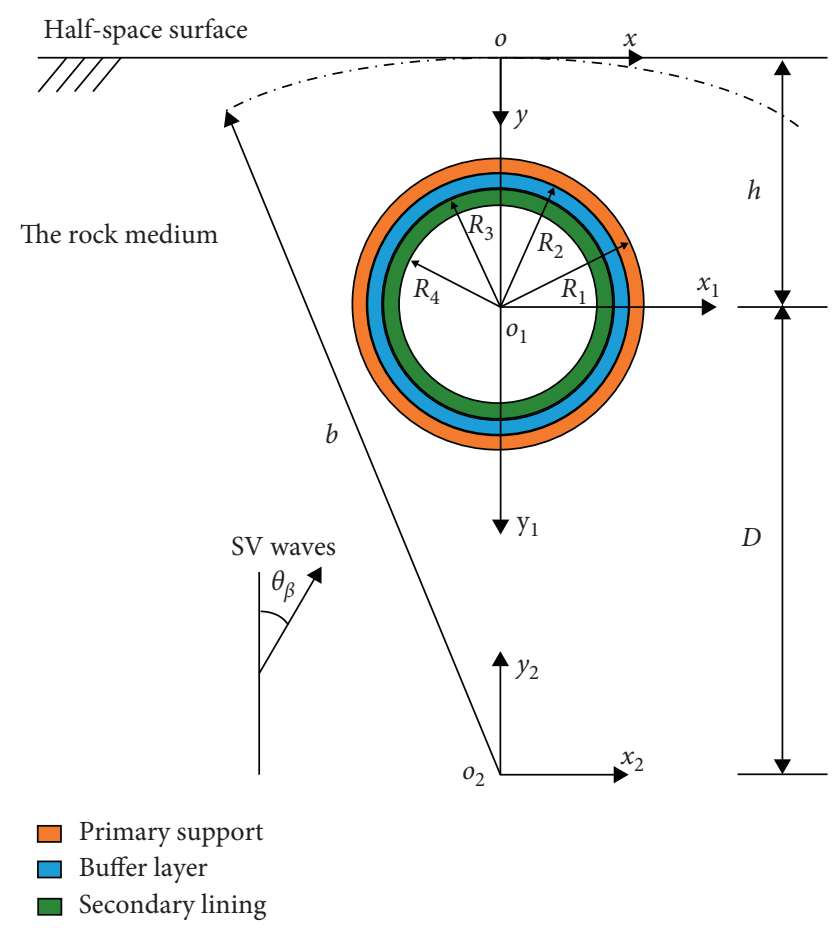

FIGURE 1: Model of composite lining with buffer layer in half-space.

2.1. Wave Function of Free Field in Half-Space. Planar harmonic SV waves are incident at $\theta_{\beta}$, regardless of the dissipation during propagation; then the displacement potential function of steady-state incident waves can be expressed in rectangular coordinate $o(x, y)$ as [30]

$$
\psi^{(i)}(x, y)=B_{0} \exp \left[i k_{s \beta}\left(x \sin \theta_{0}+y \cos \theta_{0}\right)\right] e^{-i \omega t} \text {. }
$$

Waveform transition of the incident SV waves will occur on the half-space surface, generating reflected SV and $\mathrm{P}$ waves. The displacement potential function of reflected SV waves is

$$
\psi^{(r)}(x, y)=B_{1} \exp \left[i k_{s \beta}\left(x \sin \theta_{\beta}-y \cos \theta_{\beta}\right)\right] e^{-i \omega t} .
$$

When the angle of incidence is larger than the critical angle, the reflected $\mathrm{P}$ waves will no longer be planar, but surface waves [41]. The critical angle is $\theta_{c r}=\sin ^{-1}\left(c_{\beta, s} / c_{\alpha, s}\right)$. Thus, two ways are analyzed through whether the angle of incidence is larger than the critical angle.

2.1.1. The Angle of Incidence Is Less Than the Critical Angle: $\theta_{\beta}<\theta_{c r}$. As a result, the reflected $\mathrm{P}$ waves are planar; the displacement potential function is as follows:

$$
\phi^{(r)}(x, y)=B_{2} \exp \left[i k_{s \alpha}\left(x \sin \theta_{\alpha}-y \cos \theta_{\alpha}\right)\right] e^{-i \omega t},
$$

where $B_{0}, B_{1}$, and $B_{2}$ are the amplitude of incident plane SV waves and reflected plane SV and P waves, respectively. $k_{s \alpha}=$ $\left(\omega / c_{\alpha, s}\right)$ is the number of $\mathrm{P}$ waves; $k_{s \beta}=\left(\omega / c_{\beta, s}\right)$ is the number of SV waves. $\theta_{\beta}$ is the incidence and reflected angle of SV waves, and $\theta_{\alpha}$ is the reflected angle of $\mathrm{P}$ waves. Ignoring the time item $e^{-i \omega t}$ and using Fourier-Bessel series, the displacement potential functions of the incident and reflected SV waves and reflected $\mathrm{P}$ waves in $o_{1}$, the coordinate system, are expanded as follows [30]:

$$
\begin{aligned}
\psi^{(i+r)}\left(r_{1}, \theta_{1}\right) & =\sum_{n=0}^{\infty} J_{n}\left(k_{s \beta} r_{1}\right)\left(C_{0, n} \sin n \theta_{1}+D_{0, n} \cos n \theta_{1}\right), \\
\phi^{(r)}\left(r_{1}, \theta_{1}\right) & =\sum_{n=0}^{\infty} J_{n}\left(k_{s \alpha} r_{1}\right)\left(A_{0, n} \cos n \theta_{1}+B_{0, n} \sin n \theta_{1}\right),
\end{aligned}
$$

where $J_{n}()$ is the Bessel function of the first kind and represent converging standing waves.

The coefficients $A_{0, n}, B_{0, n}, C_{0, n}$, and $D_{0, n}$ in the potential function are expressed as

$$
\begin{aligned}
\left\{\begin{array}{l}
A_{0, n} \\
B_{0, n}
\end{array}\right\}= & \varepsilon_{n} i^{n}\left\{\begin{array}{c}
\cos n \theta_{\alpha} \\
\sin n \theta_{\alpha}
\end{array}\right\} k_{1} \exp \left(i k_{s \alpha} h \cos \theta_{\alpha}\right), \\
\left\{\begin{array}{l}
C_{0, n} \\
D_{0, n}
\end{array}\right\}= & \varepsilon_{n} i^{n}\left\{\begin{array}{c}
\sin n \theta_{\beta} \\
\cos n \theta_{\beta}
\end{array}\right\}\left[\mp(-1)^{n} \exp \left(-i k_{s \beta} h \cos \theta_{\beta}\right)\right. \\
& \left.+k_{2} \exp \left(i k_{s \beta} h \cos \theta_{\beta}\right)\right],
\end{aligned}
$$

where $k_{s a} \sin \theta_{\alpha}=k_{s \beta} \sin \theta_{\beta} ; \varepsilon_{n}=1$, for $n=0 ; \varepsilon_{n}=2$, for $n \geq 1$. The values of $\varepsilon_{n}$ are derived from the Bessel function equation, which is transformed from $n=-\infty \longrightarrow \infty$ to $n=0 \longrightarrow \infty ; k_{1}$ and $k_{2}$ are the reflection coefficients of the incident wave on the half-space surface:

$$
\left.\begin{array}{c}
k_{1}=\frac{2\left(c_{p, s} / c_{s, s}\right)^{2} \sin 2 \theta_{\beta} \cos 2 \theta_{\beta}}{\sin 2 \theta_{\alpha} \cos 2 \theta_{\beta}+\left(c_{p, s} / c_{s, s}\right)^{2} \cos ^{2}\left(2 \theta_{\beta}\right)} \\
k_{2}=\frac{\sin 2 \theta_{\alpha} \cos 2 \theta_{\beta}-\left(c_{p, s} / c_{s, s}\right)^{2} \cos ^{2}\left(2 \theta_{\beta}\right)}{\sin 2 \theta_{\alpha} \cos 2 \theta_{\beta}+\left(c_{p, s} / c_{s, s}\right)^{2} \cos ^{2}\left(2 \theta_{\beta}\right)}
\end{array}\right\},
$$

where $c_{p, s}$ and $c_{s, s}$ are the wave velocities of $\mathrm{P}$ and SV in halfspace, respectively.

2.1.2. When the Angle of Incidence of Plane SV Waves Is Larger Than the Critical Angle: $\theta_{\beta}>\theta_{c r}$. The reflected angle of reflected $\mathrm{P}$ waves satisfies Snell's law: $\sin \theta_{\alpha}=$ $\left(c_{\alpha, s} / c_{\beta, s}\right) \sin \theta_{\beta}>1$; however, the reflected angle $\theta_{\alpha}$ has no real solution, thus introducing a real number $\Phi_{\alpha}$ to make the following equation hold [42]:

$$
1<\left(\frac{c_{\alpha, s}}{c_{\beta, s}}\right) \sin \theta_{\beta}=\cosh \Phi_{\alpha}=\cos i \Phi_{\alpha}=\sin \left(\frac{\pi}{2}-i \Phi_{\alpha}\right)=\sin \theta_{\alpha}
$$

where $\theta_{\alpha}=(\pi / 2)-i \Phi_{\alpha}$ is a complex angle. Defining two real numbers to simplify the equations: $k=k_{s \alpha} \sin \theta_{\alpha}=$ $k_{s \beta} \sin \theta_{s \beta} ; \gamma=-i k_{s \alpha} \cos \theta_{\alpha}$.

By transformation, the reflected $\mathrm{P}$ waves in the coordinate $(x, y)$ can be expressed as

$$
\phi^{(r)}(x, y)=B_{2} \exp (-\gamma y+i k x)
$$

which can be expressed in cylindrical coordinates, $o_{1}\left(r_{1}, \theta_{1}\right)$ : 


$$
\phi^{(r)}\left(r_{1}, \theta_{1}\right)=k_{1} \exp (-\gamma h) \exp \left(-\gamma r_{1} \cos \theta_{1}+i k r_{1} \sin \theta_{1}\right) .
$$

Further, expanding it in Fourier-Bessel series and for $\theta_{\beta}>\theta_{\text {cr }}$, it can also be solved according to the case of $\theta_{\beta}<\theta_{\text {cr }}$ :

$$
\left.\begin{array}{rl}
\phi^{(r)}\left(r_{1}, \theta_{1}\right) & =\sum_{n=0}^{\infty} J_{n}\left(k_{s \alpha} r_{1}\right)\left(A_{0, n} \cos n \theta_{1}+B_{0, n} \sin n \theta_{1}\right), \\
A_{0, n} & =\frac{1}{N} \sum_{l=0}^{2 N-1} \frac{\phi^{*}\left(r_{1},(\pi l / N)\right) \cos ((\pi l / N) n)}{J_{n}\left(k_{s a} r_{1}\right)} \\
B_{0, n} & =\frac{1}{N} \sum_{l=0}^{2 N-1} \frac{\phi^{*}\left(r_{1},(\pi l / N)\right) \sin ((\pi l / N) n)}{J_{n}\left(k_{s a} r_{1}\right)}
\end{array}\right\},
$$

When the incident SV waves and reflected SV and $\mathrm{P}$ waves in free field encounter the tunnel, radial dispersed scattering $\mathrm{P}$ waves $\phi_{s 1}\left(r_{1}, \theta_{1}\right)$ and SV waves $\psi_{s 1}\left(r_{1}, \theta_{1}\right)$ will be generated. The Fourier-Bessel series expansion of the displacement potential function is as follows:

$$
\left\{\begin{array}{l}
\phi_{s 1}\left(r_{1}, \theta_{1}\right)=\sum_{n=0}^{\infty} H_{n}^{(1)}\left(k_{s \alpha} r_{1}\right)\left(A_{s 1, n} \cos n \theta_{1}+B_{s 1, n} \sin n \theta_{1}\right), \\
\psi_{s 1}\left(r_{1}, \theta_{1}\right)=\sum_{n=0}^{\infty} H_{n}^{(1)}\left(k_{s \beta} r_{1}\right)\left(C_{s 1, n} \sin n \theta_{1}+D_{s 1, n} \cos n \theta_{1}\right),
\end{array}\right.
$$

where $H_{n}^{(1)}$ represents the Hankel functions of the first kind, which satisfies the Sommerfeld radiation condition and can represent a diverging standing wave. $A_{s 1, n}, B_{s 1, n}, \ldots$, $A_{s 1, m}, B_{s 1, m}, \ldots$ are the undetermined coefficients in the wave potential function.

In addition, the scattering $\mathrm{P}$ and $\mathrm{SV}$ waves on the tunnel surface will generate radial cohesive scattering $\mathrm{P}$ waves $\phi_{s 2}\left(r_{2}, \theta_{2}\right)$ and scattering SV waves $\psi_{s 2}\left(r_{2}, \theta_{2}\right)$ on the arc surface of half-space. The Fourier-Bessel series expansion of the displacement potential function is as follows:

$$
\left\{\begin{array}{l}
\phi_{s 2}\left(r_{2}, \theta_{2}\right)=\sum_{m=0}^{\infty} J_{m}\left(k_{s a} r_{2}\right)\left(A_{s 2, m} \cos m \theta_{2}+B_{s 2, m} \sin m \theta_{2}\right), \\
\psi_{s 2}\left(r_{2}, \theta_{2}\right)=\sum_{m=0}^{\infty} J_{m}\left(k_{s \beta} r_{2}\right)\left(C_{s 2, m} \sin m \theta_{2}+D_{s 2, m} \cos m \theta_{2}\right),
\end{array}\right.
$$

where $J_{m}()$ is the Bessel function of the first kind and $A_{s 2, n}, B_{s 2, n}, \ldots, A_{s 2, m}, B_{s 2, m}, \ldots$ are the coefficients to be determined in the wave potential function.

In summary, the total wave potential functions of SV and $\mathrm{P}$ waves in the free field are

$$
\left\{\begin{array}{l}
\phi_{s}=\phi^{r}+\phi_{s 1}+\phi_{s 2}, \\
\psi_{s}=\psi^{(i+r)}+\psi_{s 1}+\psi_{s 2} .
\end{array}\right.
$$

2.2. Wave Function of Scattering Field in Primary Support. When the P and SV waves in the free field encounter the primary support, it will lead to radial cohesive scattering $\mathrm{P}$ waves $\phi_{p 1}\left(r_{1}, \theta_{1}\right)$ and SV waves $\psi_{p 1}\left(r_{1}, \theta_{1}\right)$ and radial dispersed scattering $\mathrm{P}$ waves $\phi_{p 2}\left(r_{1}, \theta_{1}\right)$ and $\mathrm{SV}$ waves $\psi_{p 2}\left(r_{1}, \theta_{1}\right)$ in primary support:

$$
\begin{aligned}
& \left\{\begin{array}{l}
\phi_{p 1}\left(r_{1}, \theta_{1}\right)=\sum_{n=0}^{\infty} J_{n}\left(k_{p \alpha} r_{1}\right)\left(A_{p 1, n} \cos n \theta_{1}+B_{p 1, n} \sin n \theta_{1}\right) \\
\psi_{p 1}\left(r_{1}, \theta_{1}\right)=\sum_{n=0}^{\infty} J_{n}\left(k_{p \beta} r_{1}\right)\left(C_{p 1, n} \sin n \theta_{1}+D_{p 1, n} \cos n \theta_{1}\right),
\end{array}\right. \\
& \left\{\begin{array}{l}
\phi_{p 2}\left(r_{1}, \theta_{1}\right)=\sum_{n=0}^{\infty} H_{n}^{(1)}\left(k_{a_{1}} r_{1}\right)\left(A_{p 2, n} \cos n \theta_{1}+B_{p 2, n} \sin n \theta_{1}\right), \\
\psi_{p 2}\left(r_{1}, \theta_{1}\right)=\sum_{n=0}^{\infty} H_{n}^{(1)}\left(k_{\beta_{1}} r_{1}\right)\left(C_{p 2, n} \sin n \theta_{1}+D_{p 2, n} \cos n \theta_{1}\right),
\end{array}\right.
\end{aligned}
$$

where $A_{p 1, n}, B_{p 1, n}, \ldots, A_{p 2, n}, B_{p 2, n}, \ldots$ are the unknown coefficients. $k_{p \alpha}$ and $k_{p \beta}$ are the $\mathrm{P}$ and SV waves number, respectively, in the primary support. The total potential functions in the primary support are

$$
\left\{\begin{array}{l}
\phi_{p}=\phi_{p 1}+\phi_{p 2} \\
\psi_{p}=\psi_{p 1}+\psi_{p 2} .
\end{array}\right.
$$

2.3. Wave Function of Scattering Field in Buffer Layer. When $\mathrm{P}$ and SV waves in the primary support encounter the buffer layer, a transmission wave will occur at the interface. Then radial cohesive scattering $\mathrm{P}$ waves $\phi_{m 1}\left(r_{1}, \theta_{1}\right)$ and SV waves $\psi_{m 1}\left(r_{1}, \theta_{1}\right)$ and radial dispersed scattering $\mathrm{P}$ waves $\phi_{m 2}\left(r_{1}, \theta_{1}\right)$ and SV waves $\psi_{m 2}\left(r_{1}, \theta_{1}\right)$ will be led inside the buffer layer. The displacement of potential functions is

$$
\begin{aligned}
& \left\{\begin{array}{l}
\phi_{b 1}\left(r_{1}, \theta_{1}\right)=\sum_{n=0}^{\infty} J_{n}\left(k_{b \alpha} r_{1}\right)\left(A_{b 1, n} \cos n \theta_{1}+B_{b 1, n} \sin n \theta_{1}\right), \\
\psi_{b 2}\left(r_{1}, \theta_{1}\right)=\sum_{n=0}^{\infty} J_{n}\left(k_{b \beta} r_{1}\right)\left(C_{b 2, n} \sin n \theta_{1}+D_{b 2, n} \cos n \theta_{1}\right),
\end{array}\right. \\
& \left\{\begin{array}{l}
\phi_{b 2}\left(r_{1}, \theta_{1}\right)=\sum_{n=0}^{\infty} H_{n}^{(1)}\left(k_{b \alpha} r_{1}\right)\left(A_{b 2, n} \cos n \theta_{1}+B_{b 2, n} \sin n \theta_{1}\right), \\
\psi_{b 2}\left(r_{1}, \theta_{1}\right)=\sum_{n=0}^{\infty} H_{n}^{(1)}\left(k_{b \beta} r_{1}\right)\left(C_{b 2, n} \sin n \theta_{1}+D_{b 2, n} \cos n \theta_{1}\right),
\end{array}\right.
\end{aligned}
$$

where $A_{b 1, n}, B_{b 1, n}, \ldots, A_{b 2, n}, B_{b 2, n}, \ldots$ are the undetermined coefficients. $k_{b \alpha}$ and $k_{b \beta}$ are the $\mathrm{P}$ and SV waves number, respectively, in the buffer layer. Thus, the total potential functions in the buffer layer are

$$
\left\{\begin{array}{l}
\phi_{b}=\phi_{b 1}+\phi_{b 2}, \\
\psi_{b}=\psi_{b 1}+\psi_{b 2} .
\end{array}\right.
$$

2.4. Wave Function of Scattering Field in Secondary Lining. When $\mathrm{P}$ and SV waves in the buffer layer encounter the secondary lining, transmission wave will occur at the interface. Then radial cohesive scattering $\mathrm{P}$ waves $\phi_{l 1}\left(r_{1}, \theta_{1}\right)$ 
and SV waves $\psi_{11}\left(r_{1}, \theta_{1}\right)$ and radial dispersed scattering $\mathrm{P}$ waves $\phi_{12}\left(r_{1}, \theta_{1}\right)$ and SV waves $\psi_{12}\left(r_{1}, \theta_{1}\right)$ will be led inside the secondary lining. The displacement of potential functions is

$$
\begin{aligned}
& \left\{\begin{array}{l}
\phi_{l 1}\left(r_{1}, \theta_{1}\right)=\sum_{n=0}^{\infty} J_{n}\left(k_{l \alpha} r_{1}\right)\left(A_{l 1, n} \cos n \theta_{1}+B_{l 1, n} \sin n \theta_{1}\right), \\
\psi_{l 2}\left(r_{1}, \theta_{1}\right)=\sum_{n=0}^{\infty} J_{n}\left(k_{l \beta} r_{1}\right)\left(C_{l 2, n} \sin n \theta_{1}+D_{l 2, n} \cos n \theta_{1}\right),
\end{array}\right. \\
& \left\{\begin{array}{l}
\phi_{l 2}\left(r_{1}, \theta_{1}\right)=\sum_{n=0}^{\infty} H_{n}^{(1)}\left(k_{l \alpha} r_{1}\right)\left(A_{l 2, n} \cos n \theta_{1}+B_{l 2, n} \sin n \theta_{1}\right), \\
\psi_{l 2}\left(r_{1}, \theta_{1}\right)=\sum_{n=0}^{\infty} H_{n}^{(1)}\left(k_{l \beta} r_{1}\right)\left(C_{l 2, n} \sin n \theta_{1}+D_{l 2, n} \cos n \theta_{1}\right) .
\end{array}\right.
\end{aligned}
$$

where $A_{l 1, n}, B_{l 1, n}, \ldots, A_{l 2, n}, B_{l 2, n}, \ldots$ are the undetermined coefficients. $k_{l \alpha}$ and $k_{l \beta}$ are the $\mathrm{P}$ and SV waves number, respectively, in the secondary lining. Thus, the total potential functions in the secondary lining are

$$
\left\{\begin{array}{l}
\phi_{l}=\phi_{l 1}+\phi_{l 2} \\
\psi_{l}=\psi_{l 1}+\psi_{l 2}
\end{array}\right.
$$

2.5. Boundary Conditions. Based on the assumption of plane strain, there are two kinds of similar boundary conditions: (1) the radial and shear stress at the surface of half-space and the inner surface of secondary lining are zero; (2) the stress and displacement are continuous at the interface of a different medium. Thus, we can obtain the following:

(1) Zero stress at the surface of half-space:

$$
\begin{aligned}
& \sigma_{r r}^{s}=0, \\
& \tau_{r \theta}^{s}=0, \\
& \left(r_{2}=b\right) .
\end{aligned}
$$

(2) Continuous stress and displacement at the interface between primary support and half-space:

$$
\left\{\begin{array}{l}
\sigma_{r r}^{p}=\sigma_{r r}^{s}, \tau_{r \theta}^{p}=\tau_{r \theta}^{s}, \quad\left(r_{1}=R_{1}\right), \\
u_{r}^{p}=u_{r}^{s}, u_{\theta}^{p}=u_{\theta}^{s}, \quad\left(r_{1}=R_{1}\right) .
\end{array}\right.
$$

(3) Continuous stress and displacement at the interface between primary support and buffer layer:

$$
\left\{\begin{array}{l}
\sigma_{r r}^{b}=\sigma_{r r}^{p}, \tau_{r \theta}^{b}=\tau_{r \theta}^{p}, \quad\left(r_{1}=R_{2}\right), \\
u_{r}^{b}=u_{r}^{p}, u_{\theta}^{b}=u_{\theta}^{p}, \quad\left(r_{1}=R_{2}\right) .
\end{array}\right.
$$

(4) Continuous stress and displacement at the interface between buffer layer and secondary lining:

$$
\left\{\begin{array}{l}
\sigma_{r r}^{l}=\sigma_{r r}^{b}, \tau_{r \theta}^{l}=\tau_{r \theta}^{b}, \quad\left(r_{1}=R_{3}\right), \\
u_{r}^{l}=u_{r}^{b}, u_{\theta}^{l}=u_{\theta}^{b}, \quad\left(r_{1}=R_{3}\right) .
\end{array}\right.
$$

(5) Zero stress at the inner surface of the secondary lining:

$$
\begin{aligned}
\sigma_{r r}^{l} & =0, \\
\tau_{r \theta}^{l} & =0, \\
\left(r_{1}\right. & \left.=R_{4}\right),
\end{aligned}
$$

where the superscripts $s, p, b$, and $l$ correspond to the medium of half-space, primary support, buffer layer, and secondary lining, respectively, in equations (20) (24).

The dynamic stress concentration of composite lining tunnel in half-space subjected to plane SV waves is a plane strain problem. According to the potential functions of displacement and stress in elastic medium, they can be expressed as follows:

$$
\begin{aligned}
& \left\{\begin{array}{l}
u_{r}=\frac{\partial \phi}{\partial r}+\frac{1}{r} \frac{\partial \psi}{\partial \theta} \\
u_{\theta}=\frac{1}{r} \frac{\partial \phi}{\partial \theta}-\frac{\partial \psi}{\partial r}
\end{array}\right. \\
& \left\{\begin{array}{l}
\sigma_{r r}=\lambda \nabla^{2} \phi+2 \mu\left[\frac{\partial^{2} \phi}{\partial r^{2}}+\frac{\partial}{\partial r}\left(\frac{1}{r} \frac{\partial \psi}{\partial \theta}\right)\right] \\
\sigma_{\theta \theta}=\lambda \nabla^{2} \phi+2 \mu\left[\frac{1}{r}\left(\frac{\partial^{2} \phi}{\partial r^{2}}+\frac{1}{r} \frac{\partial^{2} \psi}{\partial \theta^{2}}\right)+\frac{1}{r}\left(\frac{1}{r} \frac{\partial \psi}{\partial \theta}-\frac{\partial^{2} \psi}{\partial r \partial \theta}\right)\right] \\
\tau_{r \theta}=2 \mu \frac{\partial}{\partial r}\left(\frac{1}{r} \frac{\partial \phi}{\partial \theta}\right)+\mu\left[\frac{1}{r^{2}} \frac{\partial^{2} \varphi}{\partial \theta^{2}}-r \frac{\partial}{\partial r}\left(\frac{1}{r} \frac{\partial \varphi}{\partial \theta}\right)\right]
\end{array}\right.
\end{aligned}
$$

where

$(1 / r)\left(\left(\partial^{2} \phi\right) / \partial \theta^{2}\right)$.

$\nabla^{2} \phi=\left(\left(\partial^{2} \phi\right) /\left(\partial r^{2}\right)\right)+(1 / r)\left((\partial \phi) / \partial r^{2}\right)+$

The above equations can be solved in three steps. (1) The total wave potential functions of each medium (equations (13), (15), (17), and (19)) can be substituted into equations (25) and (26); then the potential functions of stress and displacement with undetermined coefficients can be obtained. (2) The potential functions of stress and displacement obtained in the first step are substituted into the boundary conditions of the model (equations (20) (24)), a set of linear infinite series equations can be obtained, and the undetermined coefficients can be solved in MATLAB. (3) The solved coefficients are substituted back into the potential functions of stress and displacement obtained in the first step, and finally, the analytical solutions of dynamic stress in composite lining can be solved. Here, different terms are truncated for numerical calculation, and the error between adjacent calculation terms is verified. When the error is less than the preset accuracy, this term number is used as the actual convergent calculation terms. In this paper, the satisfactory results can be obtained when the number of finite terms $n$ or $m$ is 12 .

\section{Numerical Examples and Analysis Results}

The stress distribution of lining subjected to harmonic wave incidence is usually described by the dynamic stress concentration factor (DSCF), which is defined as the ratio of the maximum dynamic stress in the medium to the standard 
local stress based on the simple principle [32]. The circumferential dynamic stress concentration factor (DSCF) of composite lining can be expressed as

$$
\sigma_{\theta \theta}^{*}=\left|\frac{\sigma_{\theta \theta}}{\sigma_{\theta \theta}^{0}}\right|,
$$

where $\sigma_{\theta \theta}^{0}=k_{s \beta}^{2} G_{s}$ is the standard local stress in the surrounding rock. representing.

The geometric and mechanical parameters simultaneously determine the seismic absorption performances of buffer layer [14]. In addition, the low-frequency seismic waves have the significant influence on the underground structure [37]. Therefore, the influence of stiffness and thickness of buffer layer on composite lining subjected to low-frequency SV waves at different angles of incidence is analyzed.

\subsection{Effect of Buffer Layer Stiffness on the Dynamic Stress} Concentration Factor of Composite Lining. Young's modulus of buffer layer is a measure of the stiffness. The effect of changing Young's modulus of buffer layer on DSCF in the composite linings, when subjected to different SV waves angle of incidences, is analyzed.

First, the dimensionless frequency $(\eta)$ is defined as the ratio of inner diameter of the secondary lining to the incident wavelength [43]:

$$
\eta=\frac{2 R_{4}}{\lambda_{s \beta}},
$$

where $\lambda_{s \beta}$ is the wavelength of the incident SV waves in halfspace.

Based on existing literature [17, 41], Poisson's ratio of surrounding rocks and composite lining in half-space is 0.25 , and the velocity of compressional wave in each medium is 1.73 times that of the shear wave. The thickness of the primary support and secondary lining is $0.2 R_{4}$, the thickness ratio of the two is $2: 3$, and the thickness of the buffer layer is $0.05 R_{4}$. The dimensionless frequency $\eta$ is 0.25 . The buried depth of tunnel center $(h)$ is $2 R_{4}$. To be as accurate as possible, the radius of the great arc surface $(b)$ is $10000 R_{1}$. The angle of incidence is $0^{\circ}, 30^{\circ}, 60^{\circ}$, and $85^{\circ}$. Young's modulus ratio $\left(E_{b} / E_{s}\right)$ of buffer layer to surrounding rock is designed as $1 / 1,1 / 10$, and $1 / 50$, respectively, which represents that the relative stiffness of buffer layer decreases step by step. The analytical results of DSCF in composite lining are presented in Figure 2.

Figure 2 shows that the stiffness of buffer layer significantly affects the amplitude of DSCF in composite lining but does not obviously change the distribution at the same incident angle. When the stiffness of buffer layer is constant, the DSCF distribution of composite lining became more complex with the increase of incident angle, but the amplitude tends to decrease, indicating that the DSCF of composite lining is higher when subjected to vertical incidence than to oblique incidence, which is also verified by the existing studies [30]. The DSCF of secondary lining and primary support subjected to vertical incidence is emphatically analyzed.
First, we focus on the maximum stiffness of buffer layer $\left(E_{b} / E_{s}=1\right)$, as shown in Figure 2(a). The maximum DSCF of the secondary lining and primary support is 33.1 and 13.4, both occurring in the direction of conjugate $\pm 45^{\circ}$, which indicates that the secondary lining is bearing the main dynamic load.

Then, the stiffness reduction of buffer layer $\left(E_{b} / E_{s}=\right.$ $1 / 10$ ) is analyzed, as shown in Figure 2(b). Compared with the maximum stiffness of buffer layer $\left(E_{b} / E_{s}=1\right)$, the maximum DSCF of secondary lining decreases by $-23 \%$, while the primary support increases by $49 \%$. It suggests that the buffer layer can reduce the dynamic stress of the secondary lining, but it will increase the dynamic stress of primary support greatly. However, across the board, the DSCF of primary support and the secondary lining is close to each other. The results show that the dynamic responses of the two are consistent, which is beneficial to the coordinated deformation for the composite lining.

Continue to reduce the stiffness of buffer layer $\left(E_{b} / E_{s}=1 / 50\right)$, as shown in Figure 2(c). Compared with the maximum buffer layer stiffness $\left(E_{b} / E_{s}=1\right)$, the DSCF of secondary lining is further reduced by $-39.6 \%$, while that of primary support is further increased by $115.6 \%$. It indicates that, within a certain range, the lower the buffer layer stiffness is, the higher the primary support dynamic stress is and the lower the secondary lining dynamic stress is.

For further analyses for two reasons, on the one hand, the interaction between primary support and secondary lining will be weakened when the stiffness of buffer layer is reduced, but the interaction between primary support and surrounding rock will be strengthened. On the other hand, the reduction of the buffer layer stiffness will lead to an increase in the damping ratio, which will further increase the shock-absorbing performance in the buffer layer. Thus, it has an ideal shock-absorbing effect on the secondary lining.

In summary, changing the stiffness of the buffer layer in the composite lining plays the role of "redistributing" the seismic load. Reducing the stiffness of buffer layer is beneficial to protect secondary lining, but it will increase the dynamic stress of primary support. Therefore, the stiffness of buffer layer should not be too low. It is suggested that the stiffness ratio of buffer layer to surrounding rock is $E_{b} / E_{s}=1 / 10 \sim 1 / 50$.

\subsection{Effect of Thickness of Buffer Layer on the Dynamic Stress} Concentration Factor of Composite Lining. To explore the influence of thickness of buffer layer on the dynamic stress of composite lining, three cases were considered that the ratio of thickness of the buffer layers to inner radius of the secondary lining $\left(\left(R_{2}-R_{3}\right) / 2 R_{4}\right)$ is, respectively, $1 / 100,1 / 40$, and $1 / 20$, which indicates that the relative thickness of buffer layer increases step by step. The parameters are the same as Section 3.1. The DSCF of composite lining are shown in Figure 3.

As seen in Figure 3, at the same angle of incidence, the thickness of buffer layer significantly affects the DSCF amplitude of composite lining but does not change the distribution pattern. When the thickness of buffer layer is constant, the DSCF distribution of composite lining 


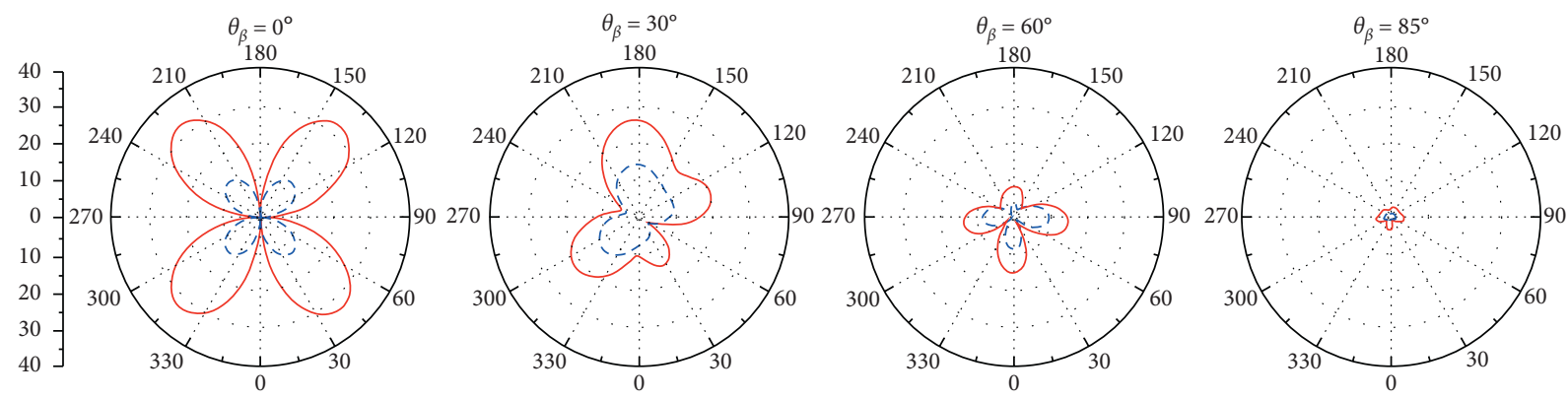

(a)
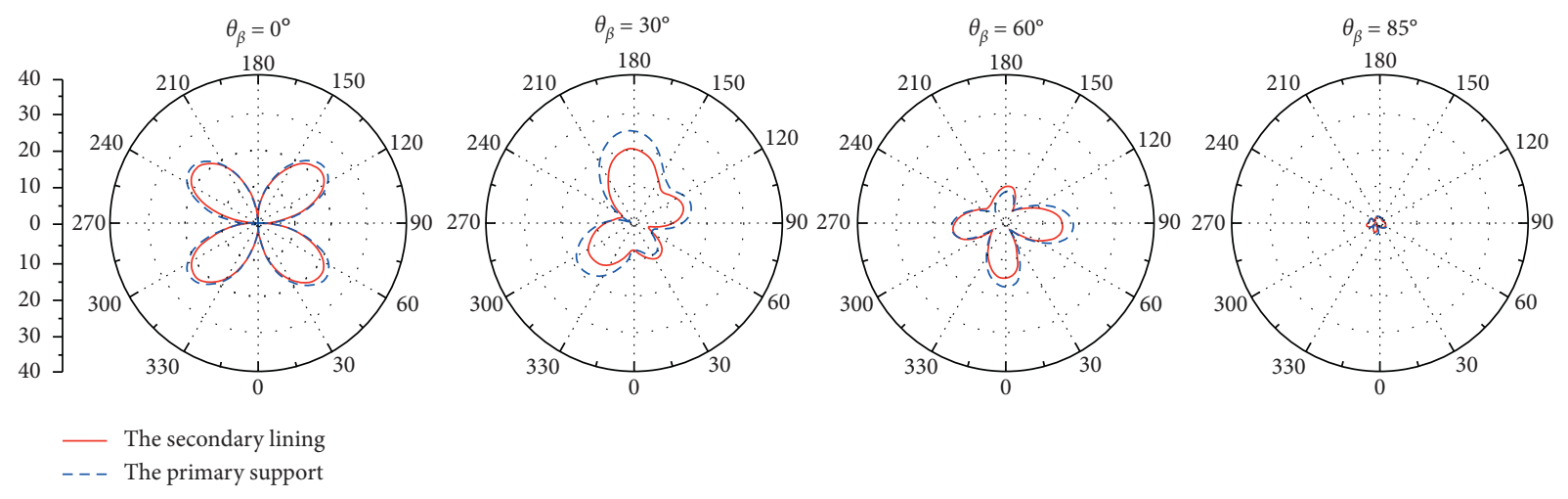

The secondary lining

(b)
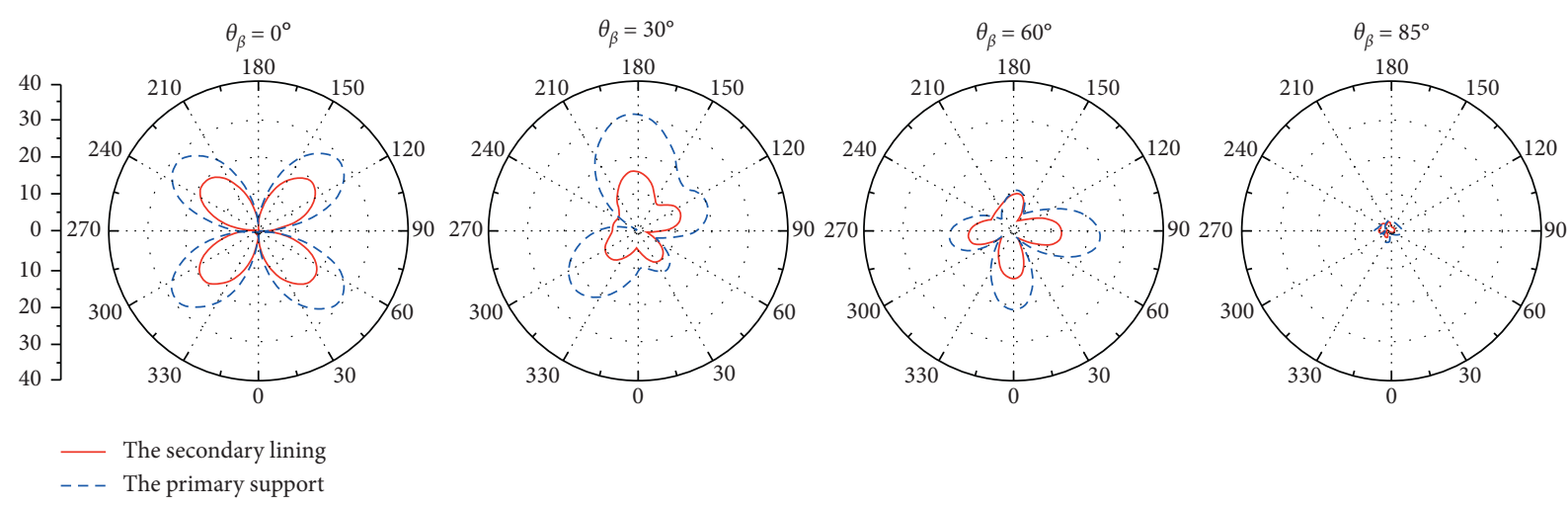

(c)

Figure 2: DSCF distribution of composite lining with different buffer layer stiffness. (a) $E_{b} / E_{s}=1$. (b) $E_{b} / E_{s}=1 / 10$. (c) $E_{b} / E_{s}=1 / 50$.

becomes more complex with the increase of incident angle, but the amplitude tends to decrease, indicating that the DSCF of composite lining subjected to vertical incidence is higher than that to oblique incidence, which is consistent with the analysis of the stiffness above.

First, we focus on the minimum thickness of buffer layer $\left(\left(R_{2}-R_{3}\right) /\left(2 R_{4}\right)=1 / 100\right)$, as shown in Figure 3(a). The maximum DSCF of the secondary lining and the primary support is 35.1 and 11.8 , both occurring in the direction of conjugate $\pm 45^{\circ}$. It suggests that the secondary lining is bearing the most of dynamic load.

Then, increasing the thickness of buffer layer $\left(\left(R_{2}-R_{3}\right) /\left(2 R_{4}\right)=1 / 40\right)$ is analyzed, as shown in Figure 3(b). Compared with the minimum thickness of buffer layer $\left(\left(R_{2}-R_{3}\right) /\left(2 R_{4}\right)=1 / 100\right)$, the maximum DSCF of secondary lining decreases by $-43 \%$, while the primary support increases by $60.7 \%$. It suggests that increasing the thickness of buffer layer can significantly change the DSCF amplitude of the composite lining in a certain range.

Further, the thickness of buffer layer increases $\left(\left(R_{2}-R_{3}\right) /\left(2 R_{4}\right)=1 / 20\right)$, as shown in Figure 3(c). Compared with the thickness of buffer layer $\left(\left(R_{2}-R_{3}\right) /\left(2 R_{4}\right)=1 / 40\right)$, the DSCF of secondary lining increased by $55 \%$, while the DSCF of primary support decreased a little. This indicates that when the thickness of buffer layer increases, the dynamic stress of secondary lining decreases first and then increases, while the dynamic stress of primary support gradually increases. Thus, it is concluded 


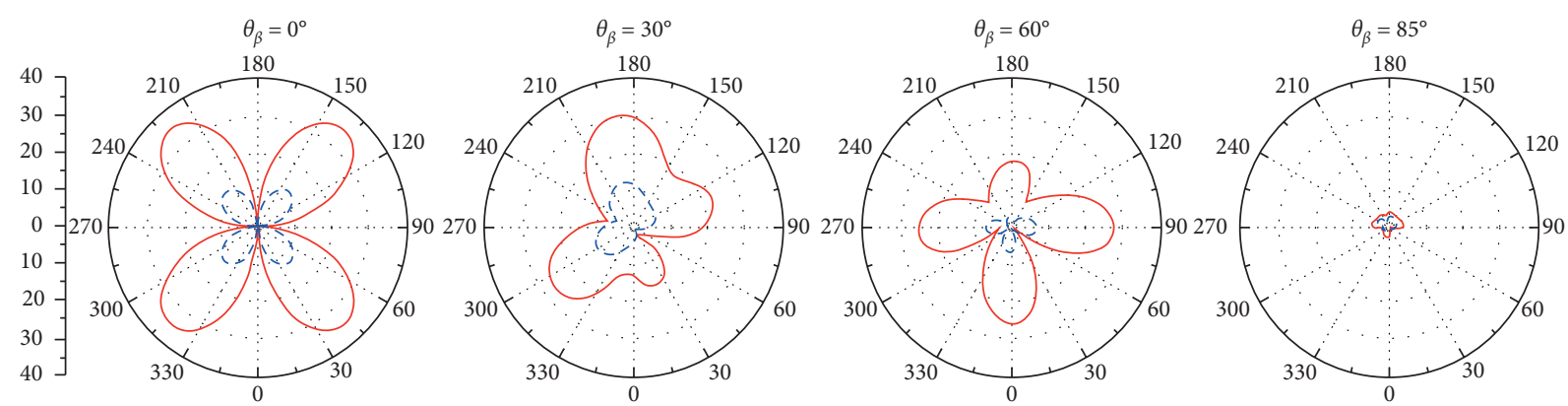

- The secondary lining

(a)
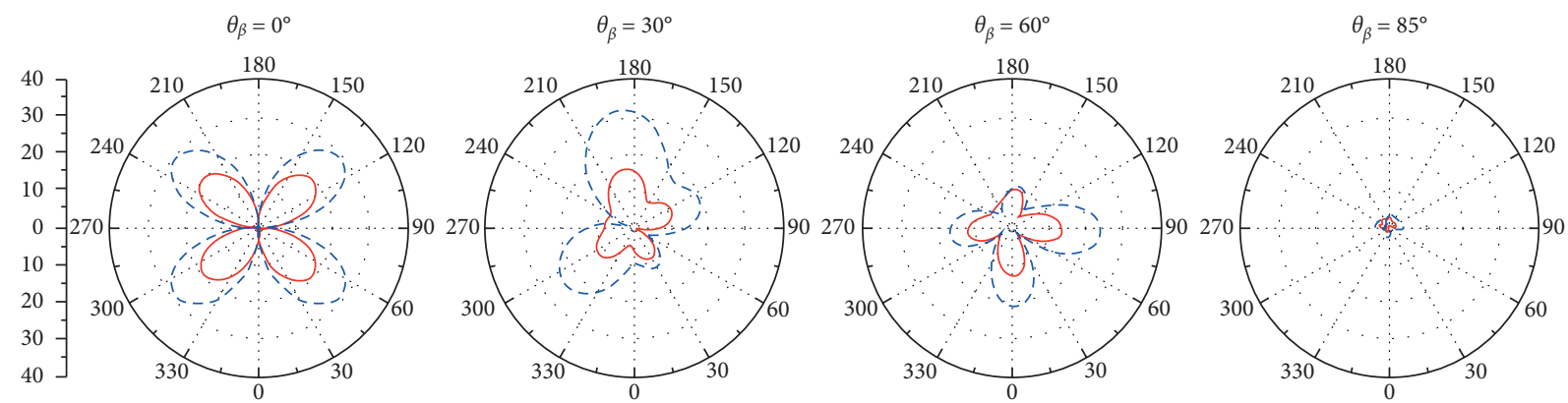

_ The secondary lining

- - - The primary support
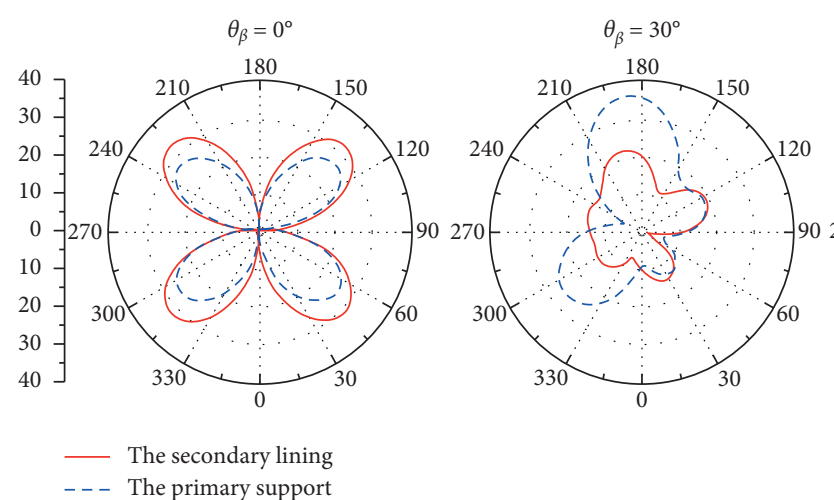

(b)

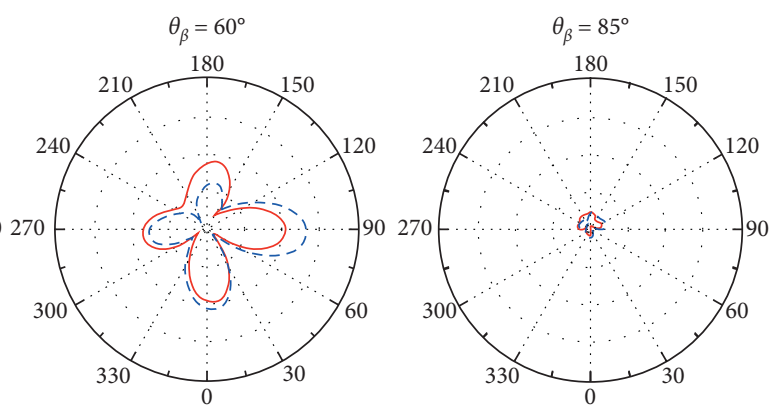

- - - The primary support

(c)

FiguRe 3: DSCF distribution of composite lining with different thickness of buffer layer. (a) $\left(R_{2}-R_{3}\right) /\left(2 R_{4}\right)=1 / 100$. (b) $\left(R_{2}-R_{3}\right) /\left(2 R_{4}\right)=$ $1 / 40$. (c) $\left(R_{2}-R_{3}\right) /\left(2 R_{4}\right)=1 / 20$.

that increasing the thickness of buffer layer does not necessarily increase the effect of shock absorption. The dynamic stress and deformation characteristics of the composite lining should be comprehensively analyzed to design the thickness of buffer layer. Based on the results, this paper will propose that the ratio of thickness of buffer layer to the inner diameter of secondary lining is $1 / 40 \sim 1 / 20$.

\section{Shaking Table Test and Numerical Simulation}

Shaking table test is undoubtedly a direct and effective method to verify the correctness and applicability of analytical solutions. Three cases of tests were conducted to explore the dynamic response of composite lining with or without buffer layer: (1) without buffer layer; (2) the thickness of buffer layer which is $1 \mathrm{~cm}$ (about $1 / 30$ of the inner diameter of secondary lining); (3) the thickness of buffer layer which is $2 \mathrm{~cm}$ (about $1 / 15$ of the inner diameter of secondary lining).

4.1. Test Facilities. The series shaking table tests were conducted at the National Engineering Laboratory for Technology of Geological Disaster Prevention in Land Transportation, Southwest Jiaotong University, as shown in Figure 4. Technical items and parameters are shown in Table 1. 


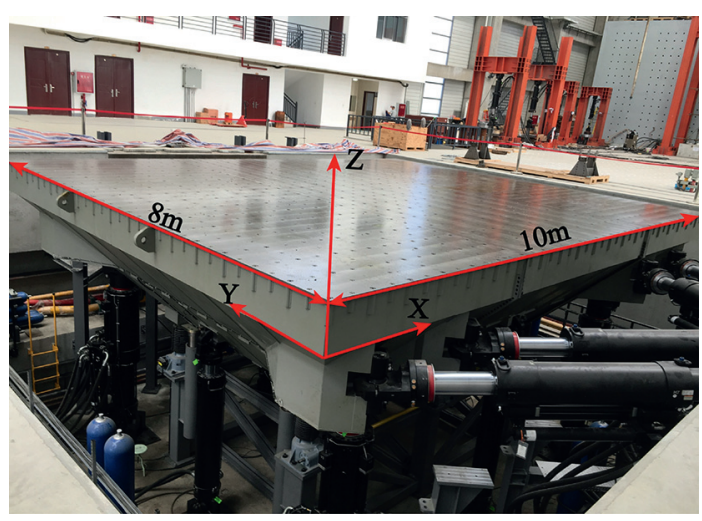

Figure 4: Shaking table $(8 \mathrm{~m} \times 10 \mathrm{~m})$.

TABLE 1: Parameters of shaking table.

\begin{tabular}{lc}
\hline Items & Parameters \\
\hline Table size (unit: $\mathrm{m} \times \mathrm{m})$ & $8.0 \times 10.0$ \\
Frequency range $(\mathrm{Hz})$ & $0.1-50$ \\
Degrees of freedom & 6 \\
Maximum weight $(t)$ & 160 \\
Maximum displacement $(\mathrm{mm})$ & Horizontal direction: \pm 800 ; vertical direction: \pm 400 \\
Maximum velocity $(\mathrm{mm} / \mathrm{s})$ & Horizontal direction: \pm 1200 ; vertical direction: \pm 1000 \\
Maximum acceleration $(\mathrm{g})$ & Horizontal direction: 1.2 ; vertical direction: 1.0 \\
Maximum overturning moment $(t \cdot \mathrm{m})$ & 600 \\
\hline
\end{tabular}

4.2. Law of Similarity and Model Materials. To describe the dynamic interaction between the composite lining and surrounding rock accurately, the similarity between the model and prototype was derived based on the Buckingham$\pi$ theorem. The basic physical quantities were geometry, Young's modulus, and density, and the other physical quantities were derived from the basic physical quantities. The similarity is shown in Table 2.

4.2.1. The Similar Materials of Tunnel Lining. The mechanical parameters of composite lining were obtained from practical engineering [44]. After a series of material tests, water, plaster, diatomite, quartz sand, and barite were adopted to simulate the composite lining with the ratio of 1 : $0.6: 0.2: 0.1: 0.4$ [45]. Table 3 lists the parameters of the lining structure for the prototype and model.

4.2.2. Similar Materials of Surrounding Rock. A mixture of fly ash, river, sand, and machine oil was selected to simulate the similar materials of surrounding rocks [44-46]. The ratio of hard rock was fly ash:river sand:oil $=50: 40: 10$, and the ratio of soft rock was fly ash:river sand:oil $=45: 40: 15$. Table 4 lists the physical parameters of the surrounding rocks for the prototype and model.

4.2.3. Similar Materials of Buffer Layer. According to the above results, when the stiffness ratio of buffer layer to surrounding rock $E_{b} / E_{s}$ was $1 / 10 \sim 1 / 50$, the shock-absorbing performance was ideal. Therefore, the optimal Young's modulus of the buffer layer was about $2 \mathrm{MPa}$, based
TABLE 2: Similarity relationship for model tests.

\begin{tabular}{lcc}
\hline Physical quantity & Similarity relation & Similarity ratio \\
\hline Length & $C_{l}$ & $1 / 30$ \\
Density & $C_{\rho}$ & $1 / 1.5$ \\
Young's modulus & $C_{E}$ & $1 / 45$ \\
Strain & $C_{\varepsilon}$ & 1 \\
Time & $C_{t}=C_{l}\left(C_{p} / C_{E}\right)^{1 / 2}$ & $1 / 5.5$ \\
Acceleration & $C_{a}=\left(C_{E} /\left(C_{l} C_{p}\right)\right)$ & 1 \\
Friction angle & $C_{\varphi}$ & 1 \\
\hline
\end{tabular}

on the similarity relationship and the mechanical parameters of similar materials. The B1 rubber sponge was selected as buffer layer, which has stable mechanical properties and was easy to process and cheap. The density was $60 \mathrm{~kg} / \mathrm{m}^{3}$, Young's modulus was $2.3 \mathrm{MPa}$, and Poisson's ratio was 0.3 , which can satisfy the similarity relationship. The fabrication process of composite lining with buffer layer is shown in Figure 5. It was mainly fabricated in four steps: (a) cutting the buffer layer according to the geometric conditions of the composite lining; (b) laying the buffer layer around the secondary lining; (c) installing the shaping formwork and wire net around buffer layer; (d) casting primary lining. The buffer layer was bonded to the composite lining by glue to achieve the rigid connection. The contact principle of the two was neither slack nor tight, so as to ensure that the buffer layer was in complete contact with the composite lining and no additional stress was generated.

4.3. Model Box Design. The model box was the container of the shaking table test, which requires sufficient strength and 
TABLE 3: Physical parameters of lining structure in the prototype and model.

\begin{tabular}{lcccc}
\hline Name & Density $\left(\mathrm{kg} / \mathrm{m}^{3}\right)$ & Young's modulus $(\mathrm{GPa})$ & Compression strength $(\mathrm{MPa})$ & Poisson's ratio \\
\hline Prototype & 2400 & 30 & 20.1 & 0.25 \\
Model & 1600 & 0.67 & 0.45 & 0.25 \\
\hline
\end{tabular}

TABle 4: Physical parameters of surrounding rock in the prototype and model.

\begin{tabular}{lccccc}
\hline Physical quantity & & Density $(\mathrm{kg} / \mathrm{m} 3)$ & Cohesion $(\mathrm{kPa})$ & Friction angle $\left(^{\circ}\right)$ & Young's modulus $(\mathrm{GPa})$ \\
\hline Hard rock & Prototype & 2100 & 135 & 38 & 10 \\
\multirow{3}{*}{ Soft rock } & Model & 1400 & 3 & 38 & 0.22 \\
& Prototype & 1700 & 50 & 27 & 1.5 \\
& Model & 1133 & 1.1 & 27 & 0.03 \\
\hline
\end{tabular}

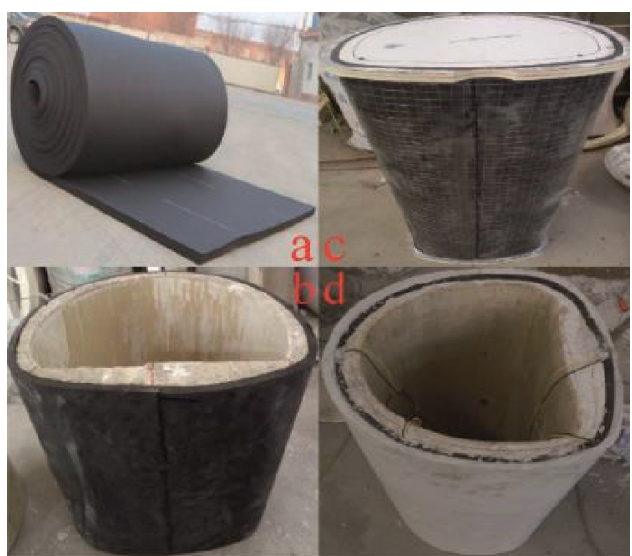

FIgURE 5: Fabrication of composite lining with buffer layer.

stiffness. The natural frequency of box should not resonate with that of the model soil and structure. The model box was made of welded steel plate and angle steel. The size of model box was $2.5 \mathrm{~m} \times 2.5 \mathrm{~m} \times 2.0 \mathrm{~m}$, as shown in Figure 6 . The sidewall of model box has a restraint effect on the soil, and the seismic wave will reflect on the sidewall for many times during shaking table test. Thus, a $10 \mathrm{~cm}$ EPS-foam was fixed on each sidewall around the model box to simulate the free-field boundary, which can absorb the reflected and scattering seismic wave to reduce the boundary effect.

4.4. Data Acquisition and Sensor Layout. Figure 7 illustrates the sensor locations. The monitoring cross sections were divided into main monitoring sections and auxiliary monitoring sections. The segments of the linings were denoted as $A, B, C$, and $D$, respectively. Four monitoring sections were designed during the test. Attention has focused on the difference of dynamic response of composite linings with or without buffer layer subjected to strong seismic wave, shown in Figures 7 (b) and 7(c).

The main monitoring sections were $2-2$ and $3-3$, which were designed to monitor the dynamic response of the composite linings in hard rock and soft rock respectively. The auxiliary monitoring sections were 1-1 and 4-4, which were designed to verify the effectiveness of the EPS-foam boundary. The sensors were mainly accelerometers and

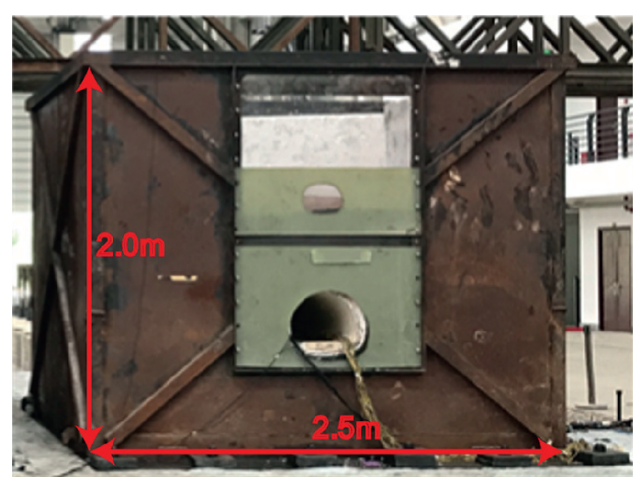

Figure 6: Model box.

strain gauges. Accelerometers $A 2$ and $A 3$ were adopted to monitor the primary support and secondary lining in hard rock, and accelerometers $A 4$ and $A 5$ were adopted to monitor the primary support and secondary lining in soft rock, respectively. A1 was designed to monitor the surface of the surrounding rock. A6 was designed to monitor the input acceleration on the shaking table. The strain gauges were stuck on each monitoring section with both the inner and outer of the linings, as illustrated in Figure 7(a).

4.5. Seismic Wave Input. The acceleration time history of the serials tests was based on the EW-component of seismic waves monitored by bedrock monitoring stations in Mao County during the Wenchuan earthquake in 2008. The peak ground acceleration (PGA) was $0.4 \mathrm{~g}$ and the duration time was compressed according to the time similarity ratio $1: 5.5$. The acceleration time history and Fourier spectrum are shown in Figure 8. The input seismic waves were horizontal shear waves perpendicular to the tunnel axis.

4.6. Boundary Effect Validation. To compare with the test data, numerical simulations of three cases were conducted with the finite difference software $\mathrm{FLAC}^{3 \mathrm{D}}$, as shown in Figure 9. The model geometry, material parameters, and monitoring points layout were the same as the tests. Solid elements were selected to simulate the surrounding rock, lining, and buffer layer. Mohr-Coulomb constitutive model was adopted for surrounding rock, and the elastic constitutive 


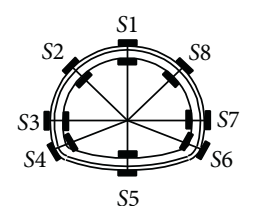

- $A$ the accelerometer

- $S$ strain gauge

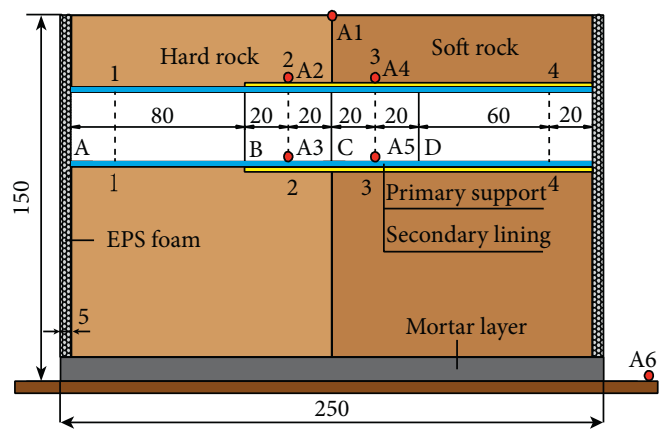

(b)

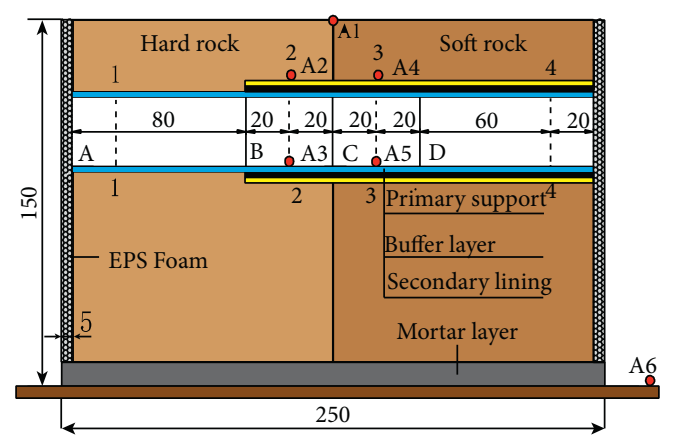

(c)

FIGURE 7: Instrumentation for seismic damage tests: (a) cross-sectional monitoring point, (b) layout of sensors in the longitudinal sections without buffer layer, and (c) layout of sensors in the longitudinal sections with a buffer layer (units: $\mathrm{cm}$ ).

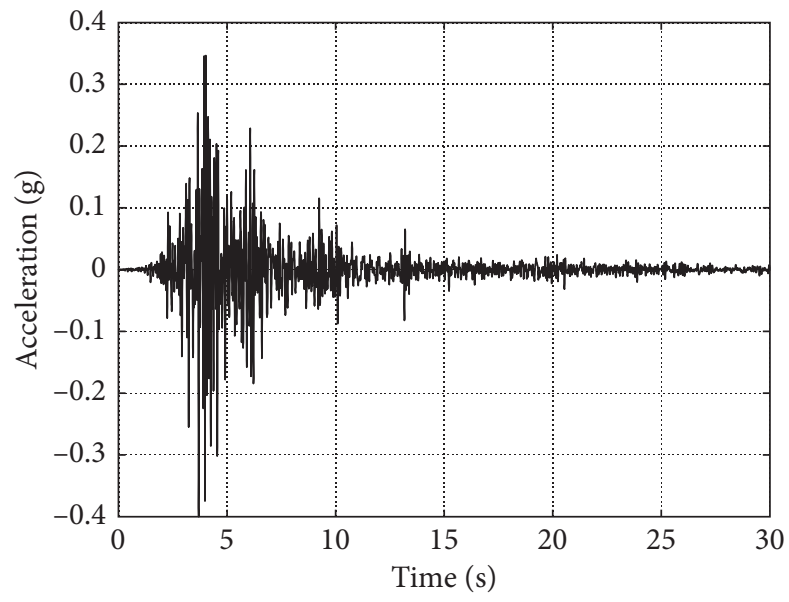

E-W component

(a)

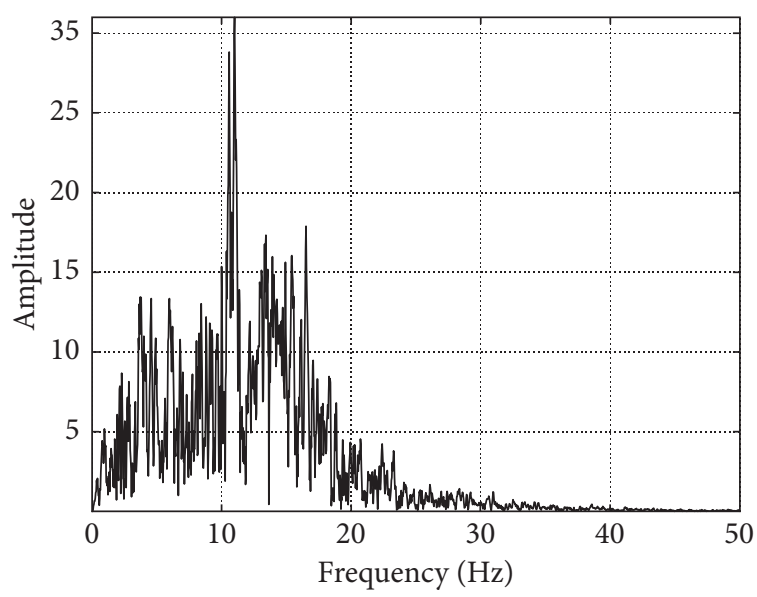

E-W component

(b)

Figure 8: (a) Acceleration time history of E-W component; (b) Fourier spectrum of E-W component.

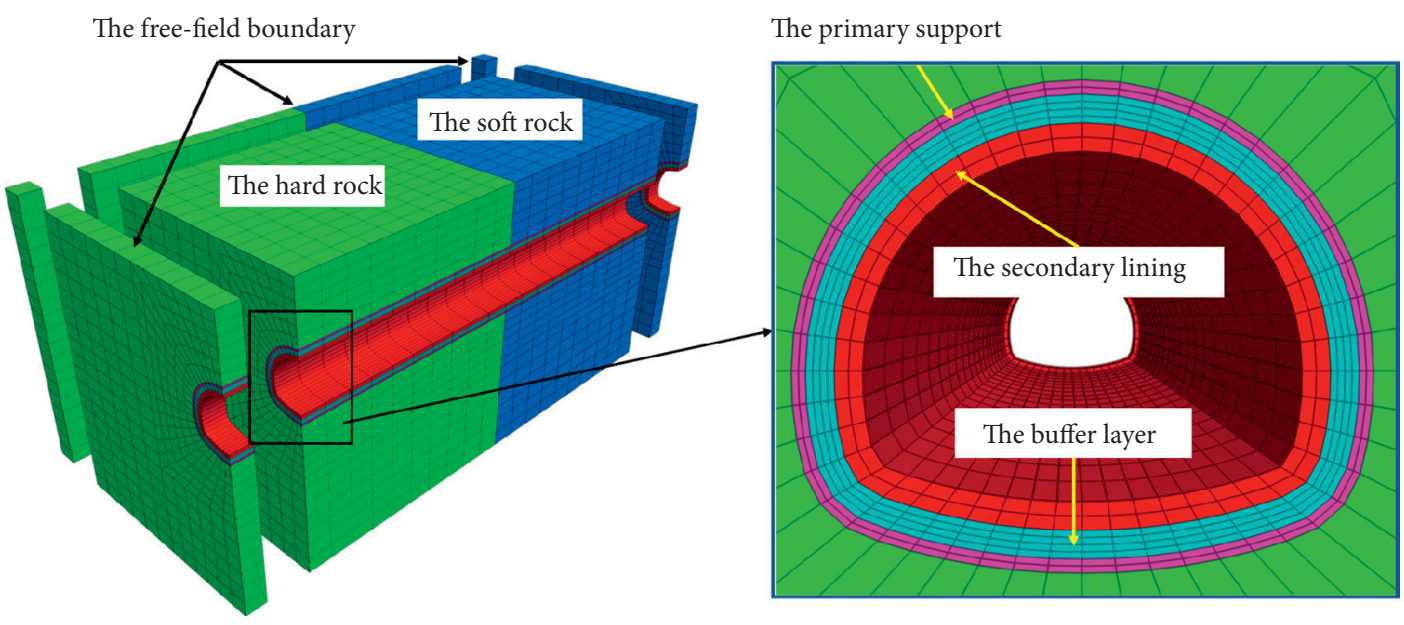

Figure 9: Numerical simulation model (1/2 model).

model was adopted for lining and buffer layer. Free-field boundary was designed and a viscous boundary was applied on the bottom. Seismic waves were perpendicular to the axial of the tunnel and realized by applying shear stress waves at the bottom of the model. Rayleigh damping was adopted, and the critical damping ratio was $5 \%$. 
Since free-field boundary was adopted in numerical simulation, the effectiveness of EPS-foam boundary can be verified by comparing the monitored acceleration spectrum of A2 and A4 in tests with the numerical simulation. As shown in Figures 10 and 11, the monitored acceleration spectrum of A2 and A4 were in good agreement with the numerical simulation, which indicated that the rigid sidewall boundary effect of the model box was effectively eliminated by the EPS-foam. It was suggested that the EPS-foam could be adopted to simulate the free-field boundary in tests.

\subsection{Acceleration Response of Composite Linings.} Figure 12 shows the acceleration time history of A3 and A5 in three cases. It can be seen that the acceleration of the secondary lining without buffer layer was the highest, followed by that when the thickness is $2 \mathrm{~cm}$, and the lowest when the thickness is $1 \mathrm{~cm}$. PGA of A3 (A5) in three cases were $0.31(0.25) \mathrm{g}>0.25(0.22) \mathrm{g}>0.15(0.17) \mathrm{g}$. It suggested that buffer layer can effectively reduce the acceleration of secondary lining, and with the increase of the thickness of buffer layer, acceleration of secondary lining first decreased and then increased. This indicated that there was an optimal interval for the thickness of buffer layer. When the thickness exceeded the optimal interval, the dynamic response of the secondary lining will be amplified to different degrees.

Figure 13 shows the peak acceleration at invert of secondary lining and crown of primary support in soft and hard surrounding rock under three cases. By comparison, when there was no buffer layer, the difference of PGA between A3 and A5 was $0.05 \mathrm{~g}$, and that between A2 and A4 was $0.06 \mathrm{~g}$. When the thickness of buffer layer was $1 \mathrm{~cm}$, the difference of PGA between A3 and A5 was $0.02 \mathrm{~g}$, and that between A2 and $A 4$ was $0.04 \mathrm{~g}$. When the thickness of buffer layer was $2 \mathrm{~cm}$, the difference of PGA between A3 and A5 PGA was $0.03 \mathrm{~g}$, and that between A2 and A4 was $0.01 \mathrm{~g}$. It is suggested that increasing the thickness of buffer layer will reduce the difference of PGA crossing the soft and hard surrounding rock and make the dynamic response of the composite lining tend to be consistent, but it will amplify the PGA.

\subsection{Dynamic Stress Concentration of Composite Linings.} The dynamic stress of composite lining was monitored by the strain gauge in the tests. The circumferential dynamic stress of each monitoring point can be calculated through Young's modulus and strains. Figure 14 shows the dynamic stress amplitude of each monitoring point of Section 3 in tests and numerical simulation.

It can be seen that the dynamic stress of secondary lining at each monitoring point was higher than that of primary support in case of no buffer layer, and the amplitude of dynamic stress at arch springing of secondary lining and spandrel of primary support was relatively high, as shown in Figure 14(a).

When the thickness of buffer layer was $1 \mathrm{~cm}$, the dynamic stress of secondary lining decreased remarkably, while the dynamic stress of primary supporting increased, especially at the arch springing. When the thickness of buffer layer was $2 \mathrm{~cm}$, the dynamic stress of the secondary lining increased on the whole, especially at the arch springing and

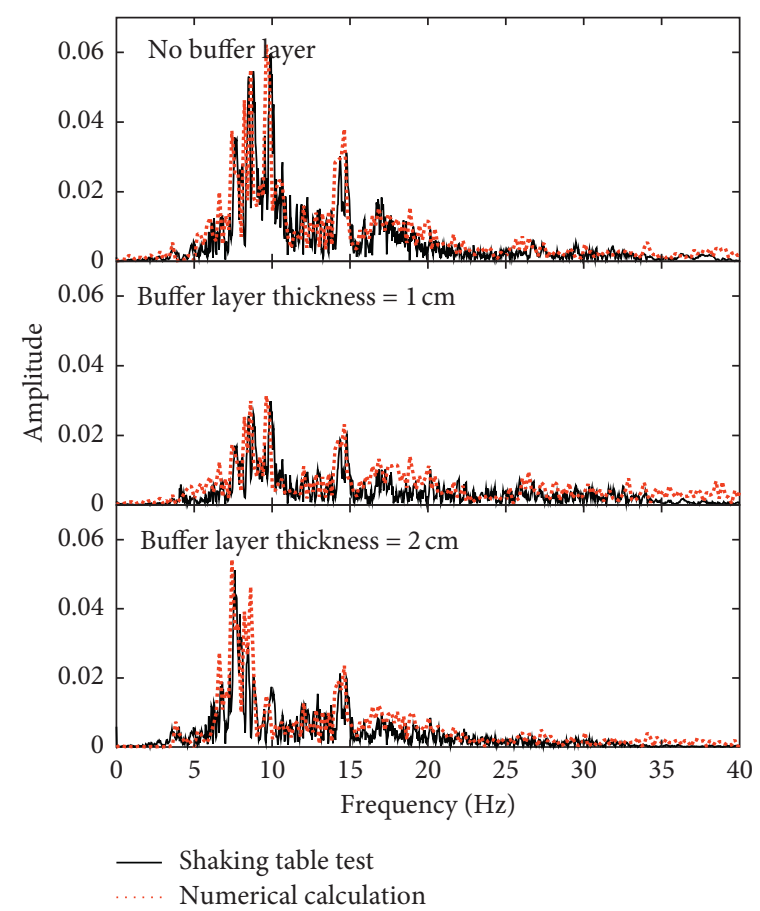

Figure 10: Acceleration spectrum of A2.

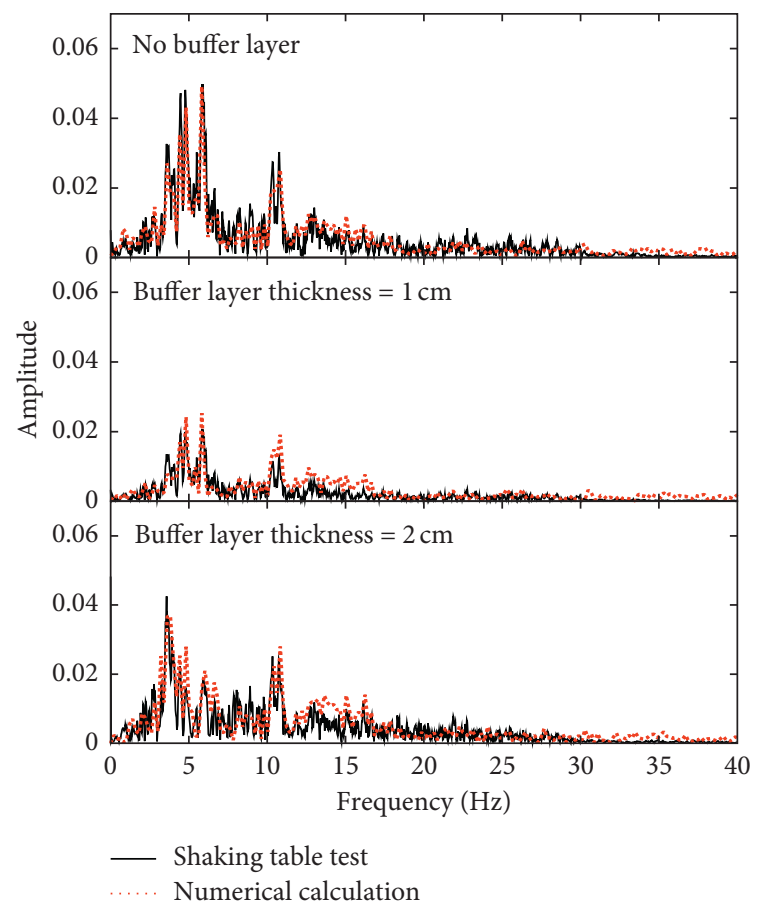

Figure 11: Acceleration spectrum of A4.

invert. The dynamic stress of the primary support was unchanged except that the crown increased dramatically. Figure 14(b) shows the results of numerical calculation, and it presented a similar rule with the test data, but it was $30 \sim 50 \%$ higher in amplitude.

According to the numerical and experimental results, the dynamic stress value of the secondary lining was $2 \sim 3$ times 


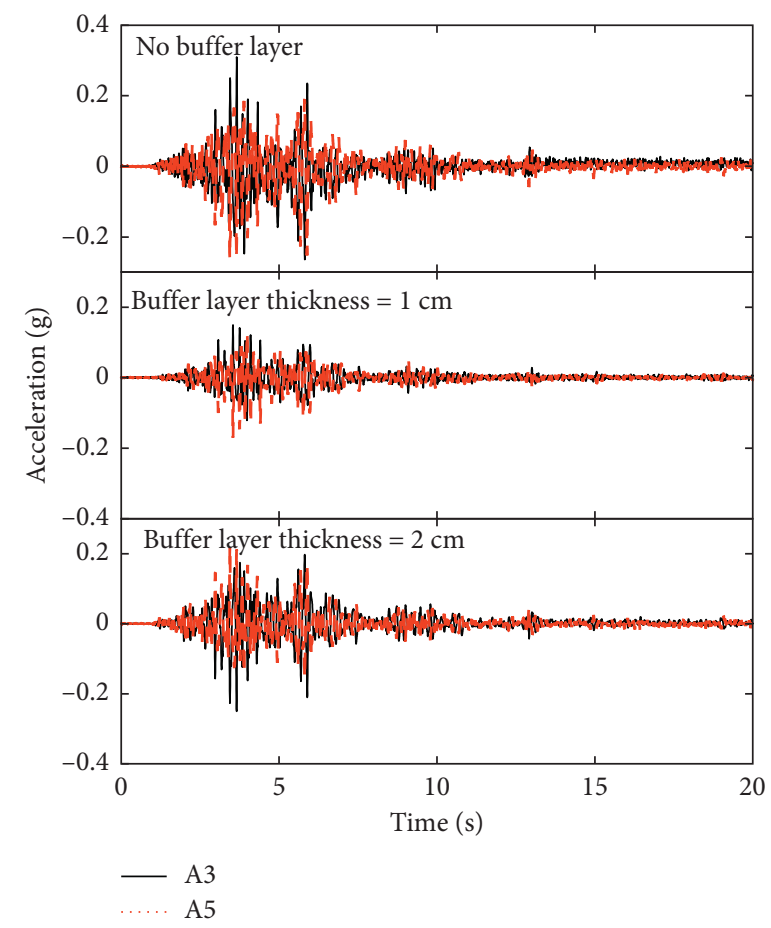

Figure 12: Acceleration time history of A3 and A5.

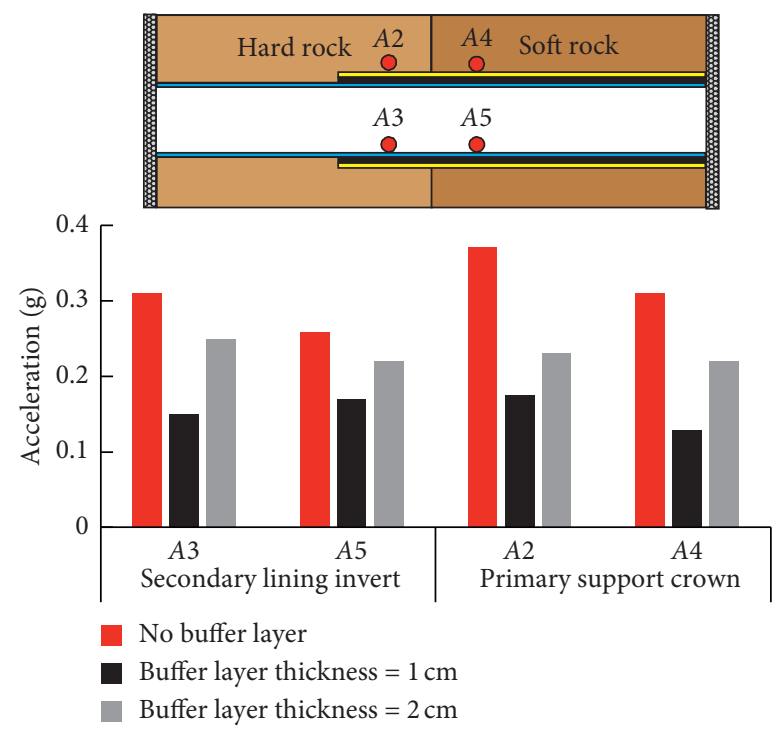

Figure 13: Contrast of peak acceleration.

that of the primary support in case of no buffer layer. Analysis of possible reasons was that "superposition effect" of incident wave and reflected wave existed on the inner surface of the secondary lining. After laying the buffer layer, on the one hand, due to the low Young's modulus and high damping ratio of the buffer layer, certain shear deformation was allowed between the primary support and the secondary lining; thus, partial energy of the seismic wave could be absorbed by the buffer layer during the strong earthquake. On the other hand, stiffness changes occurred between the primary support and the buffer layer, resulting in the "superposition effect" of the incident and reflected waves within the primary support. Therefore, the dynamic stress of the secondary lining decreased while that of the primary lining increased.

4.9. General Damage Observations. Figures 15-17 show the final cracks of composite lining after tests. In case of no buffer layer, longitudinal penetration cracks appeared in the crown, arch springing, and invert of secondary lining. Large area of falling blocks appeared in the arch springing of primary support (seen in Figure 15). 


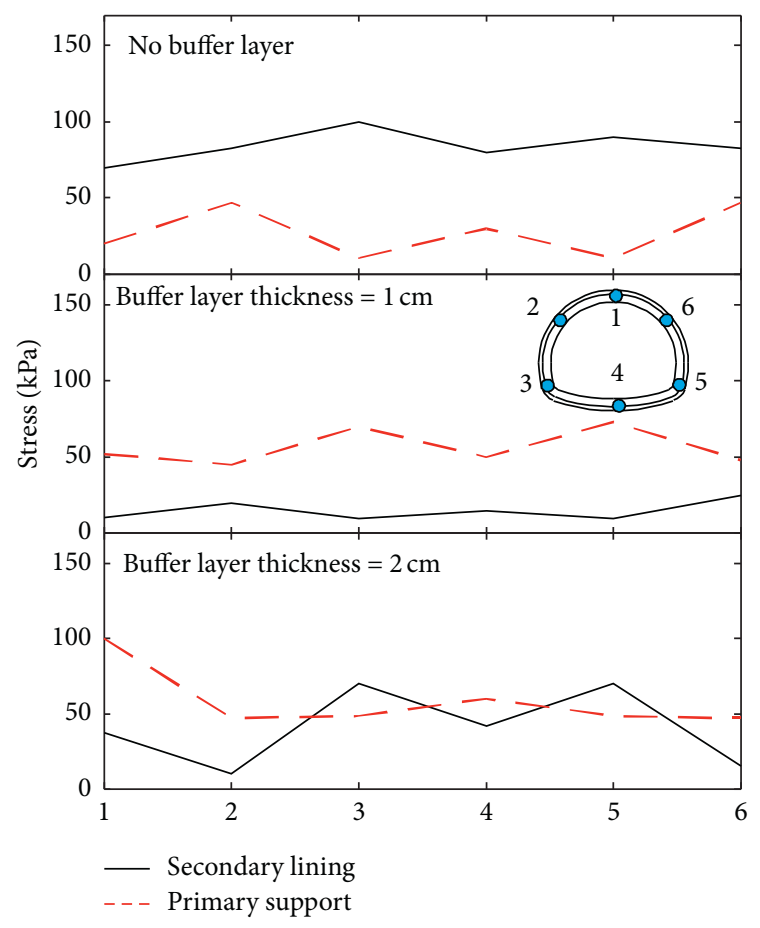

(a)

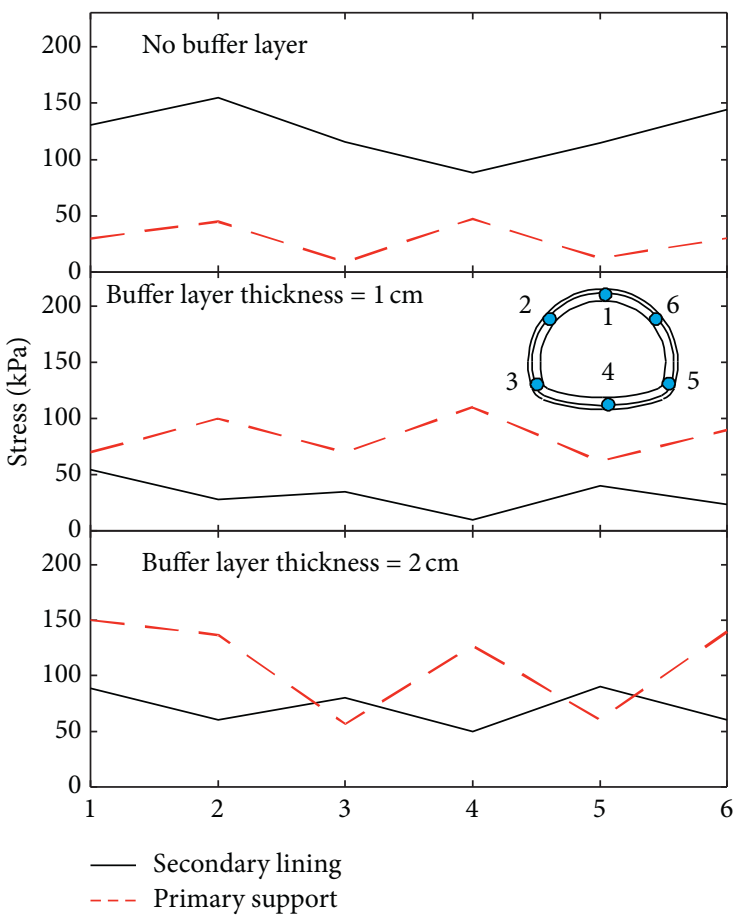

(b)

Figure 14: Dynamic stress amplitude. (a) Shaking table test. (b) Numerical simulation. 1: crown. 2: left spandrel. 3: left arch springing. 4: invert. 5: right arch springing. 6: right spandrel.

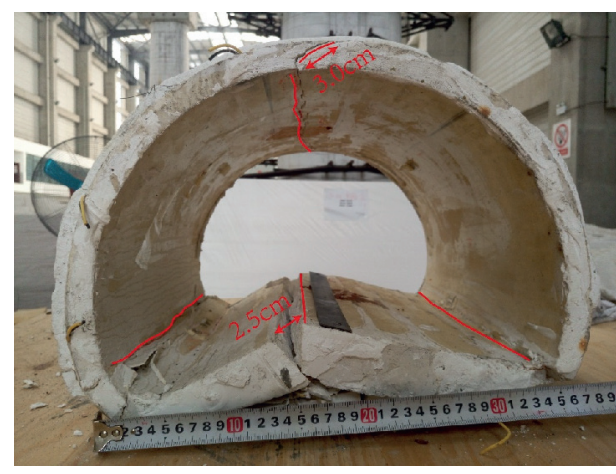

(a)

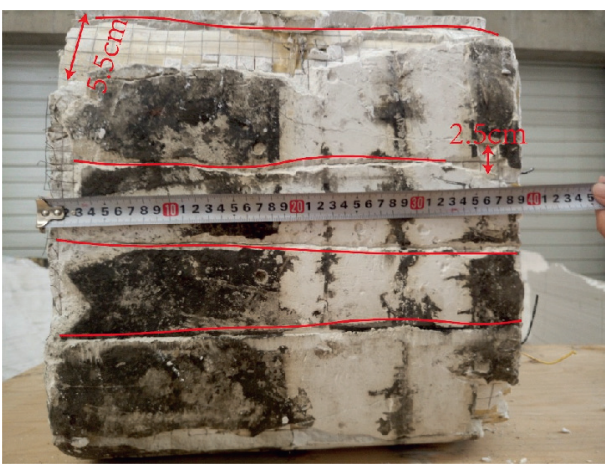

(b)

Figure 15: Damage of composite lining without buffer layer. (a) Transversal surface. (b) Longitudinal surface.

When the thickness of buffer layer was $1 \mathrm{~cm}$, the right arch springing of the secondary lining was partially cracked, the invert of the secondary lining was partially uplifted, and there was no through crack (shown in Figure 16).

When the thickness of buffer layer was $2 \mathrm{~cm}$, partial longitudinal cracks appeared at the invert and the right arch springing of secondary lining, while penetrating cracks appeared at the right arch springing of primary support (see Figure 17).

By comparing the cracks in the three cases, the crack numbers of the composite lining can be remarkably reduced with the buffer layer. When the thickness of buffer layer was
$1 \mathrm{~cm}$, the integrity of the composite lining was obviously better than that of $2 \mathrm{~cm}$. This illustrated the fact that shockabsorbing performance was weakened when the thickness of buffer layer exceeded a certain range, and it was further verified that the optimal thickness of buffer layer in the analytical solution was correct and reliable. In addition, it can be deduced from the test that the crown, arch springing, and invert of composite lining were vulnerable to be damaged during the strong ground motion. The invert was more susceptible to be damaged after laying buffer layer; therefore, the seismic design of tunnel should be paid more attention to. 


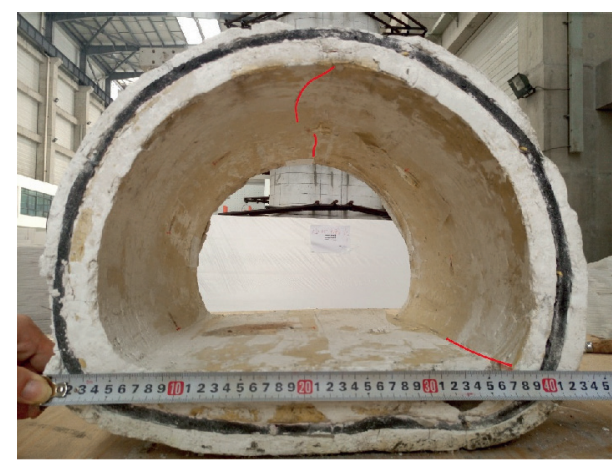

(a)

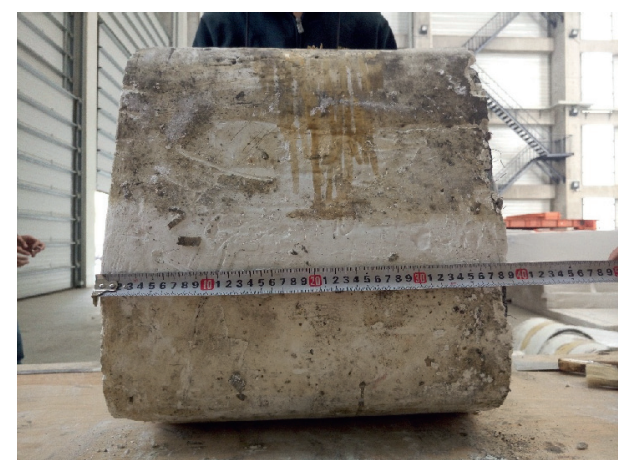

(b)

FIgURE 16: Damage of composite lining with $1 \mathrm{~cm}$ buffer layer. (a) Transversal surface. (b) Longitudinal surface.

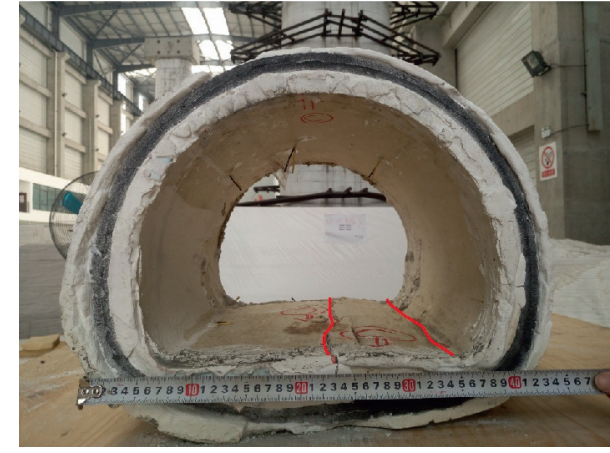

(a)

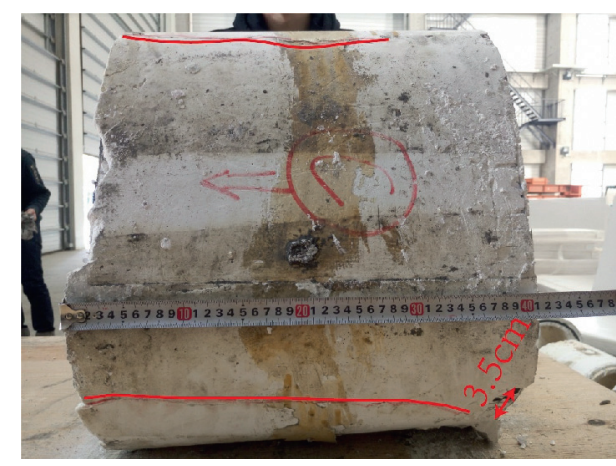

(b)

FIgURE 17: Damage of composite lining with $2 \mathrm{~cm}$ buffer layer. (a) Transversal surface. (b) Longitudinal surface.

\section{Conclusions}

Based on the Fourier-Bessel series expansion, the dynamic stress concentration factor (DSCF) of composite lining tunnel with buffer layer subjected to plane SV waves at different angle of incidences was obtained. Then, the influence of geometric and mechanical parameters of the buffer layer on composite lining was systematically analyzed, which reveals the shock-absorbing mechanism of buffer layer. Afterward, a series shaking table tests and numerical simulations were conducted to validate the reliability and correctness of analytical solutions. Finally, damage patterns of composite lining with and without buffer layer were presented. The location and size of cracks were marked on the linings, and suggestions for seismic fortification were given. These comparisons attempted to demonstrate the advantages and disadvantages of buffer layer in composite lining tunnel. The following conclusions could be drawn from this study:

(1) Increasing the incident angle of plane SV waves at low frequency, the DSCF distribution of composite lining becomes more complex than the vertical incidence, while the amplitude of DSCF decreases gradually when the stiffness and thickness of buffer layer are constant.

(2) Changing the stiffness of buffer layer will play the role of "redistributing" the seismic load. Reducing the stiffness of buffer layer is beneficial to the secondary lining, but it will increase the dynamic stress of primary support. It is suggested that the stiffness ratio of buffer layer to surrounding rock is $1 / 10 \sim 1 / 50$.

(3) Increasing the thickness of buffer layer, the dynamic stress of secondary lining decreases first and then increases, while the primary support gradually increases. Increasing the thickness of buffer layer does not necessarily achieve better shock-absorbing performance. We suggest that the ratio of buffer layer thickness to inner diameter of secondary lining is $1 / 40 \sim 1 / 20$.

(4) General damage observations show that the crown, arch springing, and invert of composite lining without buffer layer are prone to cracking during the earthquake. The invert is more susceptible to be damaged after adopting the buffer layer, which should be paid more attention to in the seismic design of composite lining tunnels.

\section{Data Availability}

The data used to support the findings of this study are included within the article. 


\section{Conflicts of Interest}

The authors declare that there are no conflicts of interest associated with this publication.

\section{Acknowledgments}

This work was supported by the National Natural Science Foundation of China (51778540, 51678501, and 51778539) and the National Key Research and Development Program of China (no.2019YFC0605104).

\section{References}

[1] Y. M. A. Hashash, J. J. Hook, B. Schmidt, and J. I-Chiang Yao, "Seismic design and analysis of underground structures," Tunnelling and Underground Space Technology, vol. 16, no. 4, pp. 247-293, 2001.

[2] T. Asakura, Y. Shiba, S. Matsuoka, T. Oya, and K. Yashiro, "Damage to mountain tunnels by earthquake and its mechanism," Doboku Gakkai Ronbunshu, vol. 659, pp. 27-38, 2000.

[3] Z. Z. Wang, Y. J. Jiang, C. A. Zhu, and T. C. Sun, "Shaking table tests of tunnel linings in progressive states of damage," Tunnelling and Underground Space Technology incorporating Trenchless Technology Research, vol. 50, pp. 109-117, 2015.

[4] H. Liu and E. Song, "Seismic response of large underground structures in liquefiable soils subjected to horizontal and vertical earthquake excitations," Computers and Geotechnics, vol. 32, no. 4, pp. 223-244, 2005.

[5] Seyyed, A. Hasheminejad, and Miri, "Seismic isolation effect of lined circular tunnels with damping treatments," Earthquake Engineering \& Engineering Vibration, vol. 7, no. 3, pp. 305-319, 2008.

[6] S. Shimamura, H. Kasai, and M. Haruumi, "Seismic isolation effect for a tunnel with a soft isolation layer," Structural Engineering Earthquake Engineering, vol. 2, no. 16, pp. 143154, 1999.

[7] H. B. Mohammad, R. M. Masoud, K. Dong-Soo, and W. C. Yun, "Effect of underground tunnel on the ground surface acceleration," Tunnelling and Underground Space Technology, vol. 44, pp. 10-22, 2014.

[8] D. S. Kim and K. Konagai, "Seismic isolation effect of a tunnel covered with coating material," Tunnelling and Underground Space Technology, vol. 15, no. 4, pp. 437-443, 2000.

[9] D.-S. Kim and K. Konagai, "Key parameters governing the performance of soft tunnel coating for seismic isolation," Earthquake Engineering \& Structural Dynamics, vol. 30, no. 9, pp. 1333-1343, 2001.

[10] K. Konagai and D.-S. Kim, "Simple evaluation of the effect of seismic isolation by covering a tunnel with a thin flexible material," Soil Dynamics and Earthquake Engineering, vol. 21, no. 4, pp. 287-295, 2001.

[11] X. Ma, F. Wang, C. Guo, and B. Sun, "Seismic isolation effect of non-water reacted two-component polymeric material coating on tunnels," Applied Sciences, vol. 10, no. 7, p. 2606, 2020.

[12] Z. Y. Chen and H. Shen, "Dynamic centrifuge tests on isolation mechanism of tunnels subjected to seismic shaking," Tunnelling and Underground Space Technology, vol. 42, pp. 67-77, 2014.

[13] J. He, W. Chen, W. Zhao, S. Huang, and Y. Yao, "Numerical test on polystyrene tunnel seismic-isolation material," Polish Journal of Chemical Technology, vol. 18, no. 3, pp. 122-127, 2016.
[14] C. L. Xin, Z. Z. Wang, and J. Yu, "The evaluation on shock absorption performance of buffer layer around the cross section of tunnel lining," Soil Dynamics and Earthquake Engineering, vol. 131, p. 106032, 2020.

[15] S. Ma, W. Chen, and W. Zhao, "Mechanical properties and associated seismic isolation effects of foamed concrete layer in rock tunnel," Journal of Rock Mechanics and Geotechnical Engineering, vol. 11, no. 1, pp. 159-171, 2019.

[16] W. Zhao, W. Chen, and D. Yang, "Interaction between strengthening and isolation layers for tunnels in rock subjected to SH waves," Tunnelling and Underground Space Technology, vol. 79, pp. 121-133, 2018.

[17] T. Li, "Damage to mountain tunnels related to the Wenchuan earthquake and some suggestions for aseismic tunnel construction," Bulletin of Engineering Geology and the Environment, vol. 71, no. 2, pp. 297-308, 2012.

[18] Y. Shen, B. Gao, X. Yang, and S. Tao, "Seismic damage mechanism and dynamic deformation characteristic analysis of mountain tunnel after Wenchuan earthquake," Engineering Geology, vol. 180, pp. 85-98, 2014.

[19] H. Yu, J. Chen, A. Bobet, and Y. Yuan, "Damage observation and assessment of the Longxi tunnel during the Wenchuan earthquake," Tunnelling and Underground Space Technology, vol. 54, pp. 102-116, 2016.

[20] C. L. Xin, Z. Z. Wang, J. M. Zhou, and B. Gao, "Shaking table tests on seismic behavior of polypropylene fiber reinforced concrete tunnel lining," Tunnelling and Underground Space Technology, vol. 88, pp. 1-15, 2019.

[21] R. J. Bathurst, S. Zarnani, and A. Gaskin, "Shaking table testing of geofoam seismic buffers," Soil Dynamics and Earthquake Engineering, vol. 27, no. 4, pp. 324-332, 2007.

[22] S. Zarnani and R. J. Bathurst, "Experimental investigation of EPS geofoam seismic buffers using shaking table tests," Geosynthetics International, vol. 14, no. 3, pp. 165-177, 2007.

[23] H. Kim, D. Kim, Y. Lee, and H. Kim, "Effect of soil box boundary conditions on dynamic behavior of model soil in $1 \mathrm{~g}$ shaking table test," Applied Sciences, vol. 10, no. 13, p. 4642, 2020.

[24] H. Xu, T. Li, L. Xia, J. X. Zhao, and D. Wang, "Shaking table tests on seismic measures of a model mountain tunnel," Tunnelling and Underground Space Technology, vol. 60, pp. 197-209, 2016.

[25] M. Geniş, "Assessment of the dynamic stability of the portals of the Dorukhan tunnel using numerical analysis," International Journal of Rock Mechanics and Mining Sciences, vol. 47, no. 8, pp. 1231-1241, 2010.

[26] G. Gazetas, N. Gerolymos, and I. Anastasopoulos, "Response of three Athens metro underground structures in the 1999 Parnitha earthquake," Soil Dynamics and Earthquake Engineering, vol. 25, no. 7-10, pp. 617-633, 2005.

[27] J. Chen, L. Jiang, J. Li, and X. Shi, "Numerical simulation of shaking table test on utility tunnel under non-uniform earthquake excitation," Tunnelling and Underground Space Technology, vol. 30, pp. 205-216, 2012.

[28] H. Yu, Y. Yuan, Z. Qiao, Y. Gu, Z. Yang, and X. Li, "Seismic analysis of a long tunnel based on multi-scale method," Engineering Structures, vol. 49, pp. 572-587, 2013.

[29] G. Wang, M. Yuan, X. Ma, and J. Wu, "Numerical study on the seismic response of the underground subway stationsurrounding soil mass-ground adjacent building system," Frontiers of Structural and Civil Engineering, vol. 11, no. 4, pp. 424-435, 2017.

[30] V. W. Lee and J. Karl, "Diffraction of SV waves by underground, circular, cylindrical cavities," Soil Dynamics and Earthquake Engineering, vol. 11, no. 8, pp. 445-456, 1992. 
[31] C. L. Xin, Z. Z. Wang, and B. Gao, "Shaking table tests on seismic response and damage mode of tunnel linings in diverse tunnel-void interaction states," Tunnelling and Underground Space Technology, vol. 77, pp. 295-304, 2018.

[32] Y. H. Pao and C. C. Mow, Diffraction of Elastic Waves and Dynamic Stress Concentrations, Crane Russak \& Company, New York, NY, USA, 1973.

[33] C. A. Davis, V. W. Lee, and J. P. Bardet, "Transverse response of underground cavities and pipes to incident SV waves," Earthquake Engineering \& Structural Dynamics, vol. 30, no. 3, pp. 383-410, 2001.

[34] V. W. LEE and M. D. TRIFUNAC, "Response of tunnels to incident SH waves," Journal of Engineering Mechanics, vol. 105, no. 4, pp. 643-659, 1979.

[35] V. W. Lee and H. Cao, "Diffraction of SV waves by circular canyons of various depths," Journal of Engineering Mechanics, vol. 115, no. 9, pp. 2035-2056, 1989.

[36] V. W. Lee, "On deformation of near a circular underground cavity subjected to incident plane P waves," European Journal of Earthquake Engineering, vol. 7, no. 1, pp. 29-35, 1993.

[37] H. Xu, T. Li, J. Xu, and Y. Wang, "Dynamic response of underground circular lining tunnels subjected to incident $\mathrm{P}$ waves," Mathematical Problems in Engineering, vol. 2014, Article ID 297424, 11 pages, 2014.

[38] H. F. Kara, "A note on response of tunnels to incident SHwaves near hillsides," Soil Dynamics and Earthquake Engineering, vol. 90, pp. 138-146, 2016.

[39] C. H. Lin, V. W. Lee, M. I. Todorovska, and M. D. Trifunac, "Zero-stress, cylindrical wave functions around a circular underground tunnel in a flat, elastic half-space: incident P-waves," Soil Dynamics and Earthquake Engineering, vol. 30, no. 10, pp. 879-894, 2010.

[40] Z. Fan, J. Zhang, and H. Xu, "Theoretical study of the dynamic response of a circular lined tunnel with an imperfect interface subjected to incident SV-waves," Computers and Geotechnics, vol. 110, pp. 308-318, 2019.

[41] X. Ji, J. Liang, and J. Yang, "On dynamic stress concentration of an underground cylindrical lined cavity subjected to incident plane P and SV waves," Journal of Tianjin University, vol. 39, no. 5, pp. 511-517, 2006.

[42] V. W. Lee and H. CAO, "Diffraction of SV waves by circular canyons of various depths," Journal of Engineering Mechanics, vol. 115, no. 9, pp. 2035-2056, 1989.

[43] J. Liang, H. Zhang, and V. W. Lee, "A series solution for surface motion amplification due to underground twin tunnels: incident SV waves," Earthquake Engineering and Engineering Vibration, vol. 2, no. 2, pp. 289-298, 2003.

[44] G. Yan, B. Gao, Y. Shen, Q. Zheng, K. Fan, and H. Huang, "Shaking table test on seismic performances of newly designed joints for mountain tunnels crossing faults," Advances in Structural Engineering, vol. 23, no. 2, pp. 248-262, 2020.

[45] G. Yan, Y. Shen, B. Gao, Q. Zheng, K. Fan, and H. Huang, "Damage evolution of tunnel lining with steel reinforced rubber joints under normal faulting: an experimental and numerical investigation," Tunnelling and Underground Space Technology, vol. 97, p. 103223, 2020.

[46] Y. S. Shen, Z. Z. Wang, J. Yu, X. Zhang, and B. Gao, "Shaking table test on flexible joints of mountain tunnels passing through normal fault," Tunnelling and Underground Space Technology, vol. 98, p. 103299, 2020. 